\title{
The formation of low-mass helium white dwarfs orbiting pulsars
}

\section{Evolution of low-mass X-ray binaries below the bifurcation period}

\author{
A. G. Istrate ${ }^{1}$, T. M Tauris ${ }^{1,2}$, and N. Langer ${ }^{1}$ \\ 1 Argelander-Institut für Astronomie, Universität Bonn, Auf dem Hügel 71, 53121 Bonn, Germany \\ e-mail: aistrate@astro.uni-bonn.de \\ 2 Max-Planck-Institut für Radioastronomie, Auf dem Hügel 69, 53121 Bonn, Germany
}

Received 25 July 2014 / Accepted 9 September 2014

\begin{abstract}
Context. Millisecond pulsars (MSPs) are generally believed to be old neutron stars (NSs) that have been spun up to high rotation rates via accretion of matter from a companion star in a low-mass X-ray binary (LMXB). This scenario has been strongly supported by various pieces of observational evidence. However, many details of this recycling scenario remain to be understood.

Aims. Here we investigate binary evolution in close LMXBs to study the formation of radio MSPs with low-mass helium white dwarf companions (He WDs) in tight binaries with orbital periods $P_{\text {orb }} \simeq 2-9 \mathrm{~h}$. In particular, we examine i) if the observed systems can be reproduced by theoretical modelling using standard prescriptions of orbital angular momentum losses (i.e. with respect to the nature and the strength of magnetic braking), ii) if our computations of the Roche-lobe detachments can match the observed orbital periods, and iii) if the correlation between WD mass and orbital period $\left(M_{\mathrm{WD}}, P_{\mathrm{orb}}\right)$ is valid for systems with $P_{\text {orb }}<2$ days.

Methods. Numerical calculations with a detailed stellar evolution code were used to trace the mass-transfer phase in $\sim 400$ close LMXB systems with different initial values of donor star mass, NS mass, orbital period, and the so-called $\gamma$-index of magnetic braking. Subsequently, we followed the orbital and the interior evolution of the detached low-mass (proto) He WDs, including stages with residual shell hydrogen burning.

Results. We find that severe fine-tuning is necessary to reproduce the observed MSPs in tight binaries with He WD companions of mass $<0.20 M_{\odot}$, which suggests that something needs to be modified or is missing in the standard input physics of LMXB modelling. Results from previous independent studies support this conclusion. We demonstrate that the theoretically calculated $\left(M_{\mathrm{WD}}, P_{\mathrm{orb}}\right)-$ relation is in general also valid for systems with $P_{\text {orb }}<2$ days, although with a large scatter in He WD masses between $0.15-0.20 M_{\odot}$. The results of the thermal evolution of the (proto) He WDs are reported in a follow-up paper (Paper II).
\end{abstract}

Key words. binaries: close - Sun: evolution - stars: neutron - white dwarfs - X-rays: binaries - accretion, accretion disks

\section{Introduction}

Millisecond pulsars (MSPs) belong to a class of radio pulsars characterized with high rotational spin rates and low magnetic fields. Most of them are observed in binary systems and it is thought that they spin rapidly because of mass accretion from a companion star in a process known as recycling (Bhattacharya \& van den Heuvel 1991; Tauris \& van den Heuvel 2006). The first MSP was discovered in 1982 (Alpar et al. 1982) and today 171 fully recycled MSPs ${ }^{1}$ are known in our Galaxy, of which 60 are found inside globular clusters and 111 are in the Galactic field. The orbital periods of binary MSP systems in the Galactic field range from $P_{\text {orb }}=93$ min to 175 days, while the companion masses can be as low as $0.02 M_{\oplus}$ (tiny planets) or as high as $\approx 1.3 M_{\odot}$ (massive white dwarfs).

MSPs in binaries can be subdivided into several classes according to the nature of their companion which can be either a degenerate or a non-degenerate object. Degenerate companions include helium white dwarfs (He WDs) and carbon-oxygen white dwarfs (CO WDs), while the non-degenerate (or semidegenerate) ones are often low-mass dwarf stars (or browndwarf like remnants) which have suffered from significant

\footnotetext{
1 According to the ATNF Pulsar Catalogue (Manchester et al. 2005), version 1.50 (June 2014). Here we define MSPs as pulsars with a spin period, $P<20 \mathrm{~ms}$. The typical measured value of the associated periodderivative is $\dot{P}<10^{-18}$.
}

mass loss and ablation from the pulsar wind (cf. the so-called black widow and redback systems in Roberts 2013 and Chen et al. 2013). The progenitors of most MSP systems are lowmass X-ray binaries (LMXBs), except for the MSPs with the more massive $\mathrm{CO} / \mathrm{ONeMg}$ WDs which are produced from intermediate-mass X-ray binaries (IMXBs) - see Tauris (2011) for a review. Pulsars with another neutron star (NS) companion are produced in high-mass X-ray binaries. Most NSs in all flavours of binary pulsar systems are produced via supernovae $(\mathrm{SNe})$ of Type Ib/c, given that their progenitors must have lost their hydrogen-rich envelopes via mass transfer in a relatively close orbit prior to the explosion.

It has been shown by Pylyser \& Savonije $(1988,1989)$ that a critical initial orbital period (the so-called bifurcation period, $\left.P_{\text {bif }}\right)$ exists at the onset of Roche-lobe overflow (RLO), separating the formation of converging LMXBs from diverging LMXBs, which shorten and widen their orbits, respectively. The theoretical estimated value of $P_{\text {bif }}^{\mathrm{RLO}}$ is $\sim 1$ day, but depends strongly on the treatment of tidal interactions and the assumed strength of magnetic braking which drains the system of orbital angular momentum (e.g. van der Sluys et al. 2005a; Ma \& Li 2009). The observed MSPs with $P_{\mathrm{orb}}>1$ day originate from relatively wide orbit LMXBs where the donor star did not fill its Roche lobe until it had evolved and expanded to become a (sub)giant, i.e. Case B RLO (e.g. Rappaport et al. 1995; Tauris \& Savonije 1999; Podsiadlowski et al. 2002). 
Table 1. Observed MSPs with He WD companions in tight circular orbits.

\begin{tabular}{|c|c|c|c|c|c|c|c|c|}
\hline Pulsar name & $\begin{array}{l}P_{\text {orb }} \\
\text { (h) }\end{array}$ & $\begin{array}{l}M_{\mathrm{WD}} \\
\left(M_{\odot}\right)\end{array}$ & $\begin{array}{l}M_{\mathrm{NS}} \\
\left(M_{\odot}\right)\end{array}$ & Eccentricity & $\begin{array}{l}P_{\text {spin }} \\
(\mathrm{ms})\end{array}$ & $\begin{array}{l}\dot{P} \\
\left(\mathrm{~s} \mathrm{~s}^{-1}\right)\end{array}$ & $\begin{array}{l}\text { WD age } \\
\text { (Gyr) }\end{array}$ & $\begin{array}{l}\text { Optical data } \\
\text { reference }\end{array}$ \\
\hline PSR J0348+0432 & 46 & 0.17 & 2.01 & $\times 10^{-6}$ & 39.1 & $2.41 \times 10^{-19}$ & $2.1 \pm 0.5$ & Antoniadis et al. (2013) \\
\hline PSR J0751+1807 & 6.31 & 0.14 & 1.34 & $7.1 \times 10^{-7}$ & 3.48 & $7.79 \times 10^{-21}$ & - & Lundgren et al. (1995) \\
\hline PSR J1738+0333 & 8.52 & 0.18 & 1.47 & $4.0 \times 10^{-6}$ & 5.85 & $2.41 \times 10^{-19}$ & - & Antoniadis et al. (2012) \\
\hline PSR J1816+4510 & 8.66 & $0.21^{a}$ & $2.0^{a}$ & $8 \times 10^{-6}$ & 3.19 & $4.31 \times 10^{-20}$ & - & Kaplan et al. (2013) \\
\hline PSR J0024-7204U & 10.3 & $0.15^{b}$ & $1.5^{b}$ & $<10^{-4}$ & 4.34 & - & 0.6 & Edmonds et al. (2001) \\
\hline PSR J1748-2446M & 10.6 & $0.17^{b}$ & $1.5^{b}$ & $<10^{-4}$ & 3.57 & - & - & \\
\hline PSR J1748-2446V & 12.1 & $0.15^{b}$ & $1.5^{b}$ & $<10^{-4}$ & 2.07 & - & - & \\
\hline PSR J0024-7204Y & 12.5 & $0.17^{b}$ & $1.5^{b}$ & $<10^{-4}$ & 2.20 & - & - & \\
\hline PSR J1641+3627D & 14.2 & $0.22^{b}$ & $1.5^{b}$ & $<10^{-4}$ & 3.12 & - & - & \\
\hline PSR J1012+5307 & 14.5 & 0.16 & 1.64 & $<8.4 \times 10^{-7}$ & 5.26 & $1.71 \times 10^{-20}$ & - & Callanan et al. (1998) \\
\hline
\end{tabular}

Notes. The top four systems have $P_{\text {orb }}<9$ h (i.e. solutions) and are found in the Galactic field. The bottom six systems are intermediate systems with $P_{\text {orb }}=9-15 \mathrm{~h}$, of which five systems are located in globular clusters. ${ }^{(a)}$ This value is very uncertain. Kaplan et al. (2013) find $M_{\mathrm{WD}} \sin ^{3} i=0.193 \pm 0.012 M_{\odot}$ and $M_{\mathrm{NS}} \sin ^{3} i=1.84 \pm 0.11 M_{\odot}$, where $i$ is the unknown orbital inclination angle. Assuming $M_{\mathrm{NS}} \leq 2.0 M_{\odot}$ yields $M_{\mathrm{WD}} \leq 0.21 M_{\odot}$. From pulsar timing (Stovall et al. 2014) a strict lower limit on the minimum companion mass is $M_{\mathrm{WD}}=0.16 M_{\odot}$ (assuming $i=90^{\circ}$ and $\left.M_{\mathrm{NS}}=1.4 M_{\odot}\right)$. ${ }^{(b)}$ For these systems we estimated $M_{\mathrm{WD}}$ by assuming $M_{\mathrm{NS}}=1.5 M_{\odot}$ and an orbital inclination angle of $60^{\circ}$.

In this work, we concentrate on investigating the evolutionary path of MSPs with He WD companions in very narrow orbits. There are four such systems known which have orbital periods, $P_{\text {orb }}<9 \mathrm{~h}$ and WD masses of $0.13<M_{\mathrm{WD}} / M_{\odot} \lesssim 0.21$. Having low-mass companions and $P_{\text {orb }}<1$ day, these systems thus descend from LMXBs in tight orbits where the donor star already initiated RLO while it was still on the main sequence (Case A RLO). As a result of their compact nature, these systems emit gravitational wave radiation and eventually evolve to become primary candidates for strong gravitational wave sources to be detected by eLISA in the $\mathrm{mHz}$ frequency range (Nelemans 2009). In Sect. 2 we list the detailed observational properties of the investigated systems. Our binary stellar evolution code is introduced in Sect. 3, and the results of the numerical calculations for the LMXBs systems are presented in Sect. 4. In Sect. 5 we discuss our findings with an emphasis on magnetic braking and the $\left(M_{\mathrm{WD}}, P_{\mathrm{orb}}\right)$-relation. Our conclusions are given in Sect. 6. In Paper II (Istrate et al. 2014) we explore the early evolution of the detached (proto) He WDs.

\section{Observational properties of MSPs with He WDs in tight orbits}

The observed properties of the binary MSPs on which we focus our attention here are described below (see also Table 1).

PSR J0348+0432 is an interesting recycled pulsar with a relatively slow spin period, $P=39.1 \mathrm{~ms}$ in a binary system with an orbital period of $P_{\text {orb }}=2.46 \mathrm{~h}$. Recently, Antoniadis et al. (2013) found that this pulsar is the most massive, precisely measured NS known with a mass of $M_{\mathrm{NS}}=2.01 \pm 0.04 M_{\odot}$, in orbit with a He WD companion of mass $M_{\mathrm{WD}}=0.172 \pm 0.003 M_{\odot}$. The estimated cooling age of the WD is about $\tau_{\text {cool }} \sim 2$ Gyr. (In an upcoming paper, Paper III, we present our analysis for the formation of this system.)

PSR J0751+1807 is an MSP with $P=3.48 \mathrm{~ms}$ in a binary system with a He WD companion and $P_{\text {orb }}=6.31 \mathrm{~h}$. Nice et al. (2008) estimated the masses of the pulsar and its companion to be $M_{\mathrm{NS}}=1.26 \pm 0.14 M_{\odot}$ and $M_{\mathrm{WD}} \simeq 0.15 M_{\odot}$, respectively. Optical and near-IR spectroscopy of the WD reveals that it has a very low (ultra-cool) effective temperature $T_{\text {eff }} \simeq 3500-4300 \mathrm{~K}$ (Bassa et al. 2006b). The cooling age of the WD is not well determined since it depends critically on residual nuclear burning in its (presumably) thick hydrogen-rich envelope. In addition, although there are no signs of pulsar irradiation, heating from the pulsar cannot be excluded.

PSR $J 1738+0333$ is another one of the handful of MSPs which have a He WD companion bright enough to make spectroscopic observations (Antoniadis et al. 2012). This system also has a very short orbital period $(8.51 \mathrm{~h})$ making it a perfect laboratory for testing theories of gravity (Freire et al. 2012). The mass of the companion is $M_{\mathrm{WD}}=0.181 \pm 0.006 M_{\odot}$ and the NS mass is constrained to be $M_{\mathrm{NS}}=1.47 \pm 0.07 M_{\odot}$.

PSR J1816+4510 is an intriguing case. It is an eclipsing MSP recently discovered by Stovall et al. (2014) who performed a radio search of a Fermi $\gamma$-ray point source. The companion star to PSR J1816+4510 ( $\left.P_{\text {orb }}=8.7 \mathrm{~h}\right)$ was detected by Kaplan et al. $(2012,2013)$ who measured an effective temperature of $T_{\text {eff }}=16000 \pm 500 \mathrm{~K}$ and estimated a companion mass of $M_{\mathrm{WD}} \sin ^{3} i=0.193 \pm 0.012 M_{\odot}$, where $i$ is the orbital inclination angle of the binary. Despite of its low surface gravity $(\log g=4.9 \pm 0.3)$ they concluded that its spectrum is rather similar to that of a low-mass He WD. For the mass of the NS they estimated $M_{\mathrm{NS}} \sin ^{3} i=1.84 \pm 0.11 M_{\odot}$. Assuming that $M_{\mathrm{NS}} \leq 2.0 M_{\odot}$ (i.e. less than the highest precisely measured NS mass known to date) this yields $M_{\mathrm{WD}} \leq 0.21 M_{\odot}$. As we discuss in Sect. 4.5, however, based solely on its $P_{\text {orb }}$, combined with modelling of the orbital period evolution of LMXBs, we would even expect $M_{\mathrm{WD}} \lesssim 0.18 M_{\odot}$. (See also Paper II for further discussions on the nature of this companion star.)

In addition to these four systems, there are a number of MSPs with low-mass He WD companions and slightly larger $P_{\text {orb }}=9-15$ h. Five of these MSPs are found in globular clusters. Usually, binary MSPs observed in dense environments like globular clusters are excluded from comparison to theoretical modelling of binaries because of the possibility that the observed MSP companion was exchanged via an encounter event. However, there are some MSPs found in globular clusters which have very small eccentricities $\left(e<10^{-4}\right.$, Paulo Freire, priv. comm.). This we take as good evidence that the present He WD companion is indeed the one which was the former donor star in the LMXB phase, and thus responsible for recycling the MSP. Therefore, we include these five MSPs in Table 1 as well.

Finally, we note that a number of low-mass He WDs $\left(\leq 0.20 M_{\odot}\right)$ with $P_{\text {orb }}<15 \mathrm{~h}$ are also found in double WD systems (e.g. Kaplan et al. 2014, and references therein). These 
WDs often have a massive CO WD companion and evolved via stable RLO in cataclysmic variable (CV) systems. Although the structure and the properties of these low-mass He WDs are similar to the ones with radio pulsar companions, we restrict ourselves to the latter sources in this work (see, however, Paper II for further discussions of these systems).

To summarize, the systems described above all have similar properties: their $P_{\text {orb }}$ is very short (in the range of $2-15 \mathrm{~h}$ ), and the He WD companions have typical masses of $0.14-0.18 M_{\odot}$. Given these characteristics, in this work we explore their formation paths (with a special focus on the systems with $P_{\text {orb }}<9$ h) and discuss the underlying physical assumptions of the applied standard modelling for loss of orbital angular momentum via magnetic braking.

\section{Numerical methods and physical assumptions}

We consider as a starting point binary systems which consist of a NS orbiting a low-mass main-sequence star. Such systems are expected to have formed from zero-age main sequence (ZAMS) binaries with a massive $\left(\sim 10-25 \quad M_{\odot}\right)$ primary star and a low-mass $\left(\sim 1-2 \quad M_{\odot}\right)$ companion in a relatively close orbit, and which subsequently survived a common-envelope phase, followed by a supernova explosion (Bhattacharya \& van den Heuvel 1991; Tauris \& van den Heuvel 2006). Numerical calculations with a detailed stellar evolution code were then used in this study to trace the mass-transfer phase in roughly 400 close LMXB systems with different initial values of donor star mass, NS mass, orbital period and the so-called $\gamma$ index of magnetic braking. Subsequently, we followed the evolution of the low-mass (proto) He WD, including stages with residual hydrogen shell burning.

We used the BEC-code which is a binary stellar evolution code originally developed by Braun (1997) on the basis of a single-star code (Langer 1998, and references therein). It is a one-dimensional implicit Lagrangian code which solves the hydrodynamic form of the stellar structure and evolution equations (Kippenhahn \& Weigert 1990). The evolution of the donor star, the mass-transfer rate, and the orbital separation are computed simultaneously through an implicit coupling scheme (see also Wellstein \& Langer 1999) using the Roche-approximation in the formulation of Eggleton (1983). To compute the mass-transfer rate, the code uses the prescription of Ritter (1988). It employs the radiative opacities of Iglesias \& Rogers (1996), which are interpolated in tables as a function of density, temperature, and chemical element mass fractions, including carbon and oxygen. For the electron conduction opacity, the code follows Hubbard \& Lampe (1969) in the non-relativistic case, and Canuto (1970) in the relativistic case. The stellar models are computed using extended nuclear networks including the PP I, II, and III chains and the four CNO-cycles. Chemical mixing due to convection, semiconvection and overshooting is treated as a diffusion process. Thermohaline mixing is also included in the code (cf. Cantiello \& Langer 2010), whereas gravitational settling and radiative levitation is not. Finally, the accreting NS is treated as a point mass.

A slightly updated version of this code for LMXBs and IMXBs has recently been applied to study the formation of MSPs (Tauris et al. 2011, 2012, 2013; Lazarus et al. 2014). In our models we assumed a mixing-length parameter of $\alpha=l / H_{\mathrm{p}}=$ 2.0 and a core convective overshooting parameter of $\delta_{\mathrm{ov}}=0.10$. Tauris et al. (2013) recently tested several models of wide-orbit LMXB evolution using $\alpha=l / H_{\mathrm{p}}=1.5$ which resulted in only slightly smaller final WD masses $(\sim 1 \%)$, orbiting recycled pulsars in somewhat closer orbits (up to $\sim 3 \%$ decrease in $P_{\text {orb }}$ ). The magnetic braking was implemented as outlined below.

\subsection{Orbital angular momentum treatment}

We considered the change in orbital angular momentum,

$\frac{\dot{J}_{\text {orb }}}{J_{\text {orb }}}=\frac{\dot{J}_{\mathrm{ml}}}{J_{\text {orb }}}+\frac{\dot{J}_{\mathrm{gwr}}}{J_{\text {orb }}}+\frac{\dot{J}_{\mathrm{mb}}}{J_{\text {orb }}}$,

with contributions from mass loss, gravitational wave radiation, and magnetic braking, respectively.

\subsubsection{Loss of orbital angular momentum due to mass loss}

We solved the orbital angular momentum balance equation (e.g. Eq. (20) in Tauris \& van den Heuvel 2006) using the isotropic re-emission model (Bhattacharya \& van den Heuvel 1991; van den Heuvel 1994; Tauris 1996; Soberman et al. 1997). In this model matter flows over from the donor star $\left(M_{2}\right)$ to the accreting NS $\left(M_{\mathrm{NS}}\right)$ in a conservative manner and thereafter a certain fraction, $\beta$ of this matter is ejected from the vicinity of the NS with the specific orbital angular momentum of the NS. Hence, one can express the loss of orbital angular momentum due to mass loss as

$\mathrm{d} J_{\mathrm{ml}}=\frac{J_{\mathrm{NS}}}{M_{\mathrm{NS}}} \beta \mathrm{d} M_{2}=\frac{\mu}{M_{\mathrm{NS}}^{2}} J_{\text {orb }} \beta \mathrm{d} M_{2}$

or

$\frac{\dot{J}_{\mathrm{ml}}}{J_{\mathrm{orb}}}=\frac{\mu}{M_{\mathrm{NS}}^{2}} \beta \dot{M}_{2}=\frac{\beta q^{2}}{1+q} \frac{\dot{M}_{2}}{M_{2}}$,

where $\mu=M_{\mathrm{NS}} M_{2} /\left(M_{\mathrm{NS}}+M_{2}\right)$ is the reduced mass, and $q=$ $M_{2} / M_{\text {NS }}$ denotes the ratio between donor star mass and the mass of the NS accretor. Keep in mind that a fraction $1-\beta$ of the matter lost from the donor star is accreted onto the NS. The rate of wind mass loss from the low-mass donor star is negligible compared to the mass-loss rate via RLO.

\subsubsection{Loss of orbital angular momentum due to gravitational wave radiation}

The second term on the right-hand side of Eq. (1) gives the loss of orbital angular momentum due to gravitational wave radiation (Landau \& Lifshitz 1971),

$\frac{\dot{J}_{\mathrm{gwr}}}{J_{\text {orb }}}=-\frac{32 G^{3}}{5 c^{5}} \frac{M_{2} M_{\mathrm{NS}} M}{a^{4}}$,

where $G$ is the gravitational constant, $c$ is the speed of light in vacuum, $a$ is the orbital separation, and $M=M_{\mathrm{NS}}+M_{2}$ is the total mass of the system. The validity of this mechanism has been beautifully demonstrated in PSR $1913+16$, which is considered as an ideal gravity laboratory (e.g. Weisberg et al. 2010). For sufficiently narrow orbits the above equation becomes the dominant term in Eq. (1), causing $a$ to decrease. Gravitational wave radiation is the major force driving the mass transfer in very narrow binaries, such as CVs (below the period gap) and ultra-compact X-ray binaries (Faulkner 1971; van Haaften et al. 2012). Therefore, the orbits of very narrow LMXBs will tend to continuously shrink (i.e. converging systems) until a period minimum is reached, before hydrogen burning is exhausted and the donor star becomes fully degenerate (Paczynski \& Sienkiewicz 1981; Rappaport et al. 1982; Nelson et al. 1986; Podsiadlowski et al. 2002; Lin et al. 2011). At this point the donor star has a 
mass of typically $M_{2} \approx 0.05-0.07 M_{\odot}$ and $P_{\text {orb }} \approx 40-80 \mathrm{~min}$. The subsequent evolution causes the orbit to widen because of the extreme mass ratio between the small donor star mass and the accreting NS. According to modelling of LMXBs (e.g. Benvenuto et al. 2012; Chen et al. 2013), the subsequent ablation of the donor star from the pulsar wind leads to the production of the so-called black widow pulsars which have companion star masses of a few $0.01 M_{\odot}$ and $P_{\text {orb }} \approx 2-10 \mathrm{~h}$ (Fruchter et al. 1988; Stappers et al. 1996; Roberts 2013). These systems may eventually form pulsar planets (Wolszczan \& Frail 1992) or become isolated MSPs (Backer et al. 1982).

\subsubsection{Loss of orbital angular momentum due to magnetic braking}

In synchronized binaries with low-mass stars $\left(\lesssim 1.5 M_{\odot}\right)$, the loss of spin angular momentum due to a magnetic wind occurs at the expense of the orbital angular momentum (e.g., Mestel 1968; Verbunt \& Zwaan 1981). However, a fundamental law of angular momentum loss is unknown for rapidly rotating stars. To compute the angular momentum loss due to magnetic braking, we adopt the prescription of Rappaport et al. (1983),

$\frac{\dot{J}_{\mathrm{MB}}}{J_{\mathrm{orb}}}=-3.8 \times 10^{-30} f \frac{R_{\odot}^{4}\left(R_{2} / R_{\odot}\right)^{\gamma} G M^{2}}{a^{5} M_{\mathrm{NS}}} \mathrm{s}^{-1}$,

where $R_{2}$ is the radius of the donor star, $f$ is scaling factor (of the order of unity) and $\gamma$ is the magnetic braking index. Here, we have investigated the effect of systematically applying various values of $\gamma$ between 2 and 5. Larger values of $\gamma$ seem to produce too strong magnetic braking compared to observations of lowmass stars in open clusters (cf. Sect. 5.1 where we discuss the nature of the magnetic braking law).

The net effect of applying the above prescription for magnetic braking is that the orbital period of close binaries $\left(P_{\text {orb }} \simeq\right.$ 2-5 days) typically decreases by a factor of three (depending on $\gamma$ ) prior to the RLO, i.e. magnetic braking causes orbital decay and forces the donor star to fill its Roche lobe and initiate mass transfer already on the main sequence or early into the Hertzsprung gap.

To optimize the analysis of our investigation and to enable us to better interpret the results in a coherent manner, we have simply assumed magnetic braking to operate in all our binaries (which have donor star masses $1.1 \leq M_{2} / M_{\odot} \leq 1.6$ ) at all times between the ZAMS and until the end of our calculations.

\subsection{Mass accretion rate and accretion efficiency}

The accretion rate onto the NS is assumed to be Eddington limited and is given by

$\dot{M}_{\mathrm{NS}}=\left(\min \left[\left|\dot{M}_{2}\right|, \dot{M}_{\mathrm{Edd}}\right]\right) e_{\mathrm{acc}} k_{\mathrm{def}}$

where $e_{\mathrm{acc}}$ is the fraction of matter transferred to the NS which actually ends up being accreted and remains on the NS, and $k_{\text {def }}$ is a factor that expresses the ratio of gravitational mass to rest mass of the accreted matter (depending on the equation-ofstate of supranuclear matter, $k_{\mathrm{def}} \simeq 0.85-0.90$; e.g. Lattimer \& Prakash 2007). The accretion efficiency of MSPs formed in LMXBs has been shown to be about $30 \%$ in several cases (Tauris \& Savonije 1999; Jacoby et al. 2005; Antoniadis et al. 2012; Tauris \& van den Heuvel 2014), even in close systems where the mass-transfer rate is expected to be sub-Eddington $\left(\left|\dot{M}_{2}\right|<\dot{M}_{\text {Edd }}\right)$ at all times. Hence, as a default value we assumed

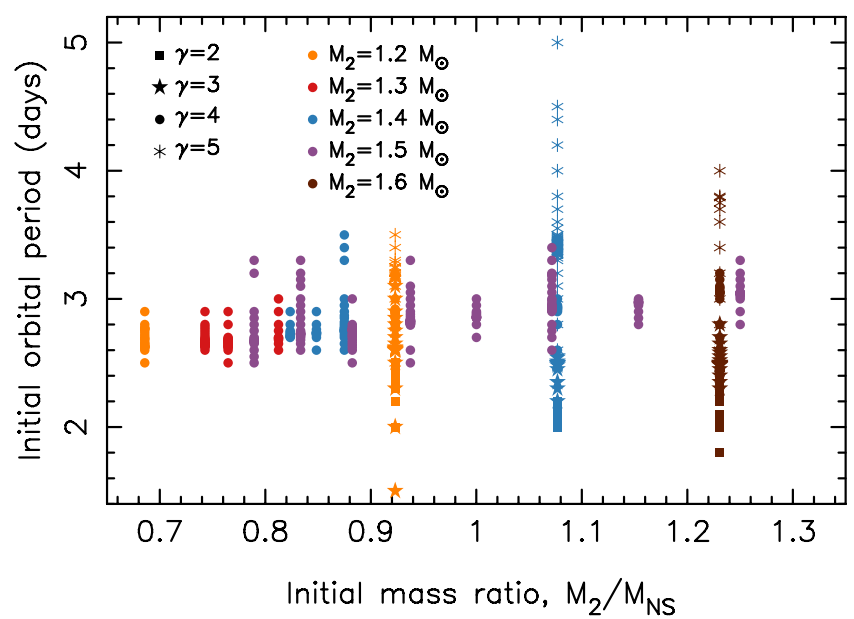

Fig. 1. Grid of initial parameters for the studied LMXB configurations, yielding a total of more than 400 models. The colours correspond to different donor star masses $\left(M_{2}\right)$, and the various symbols indicate different values of the magnetic braking index, $\gamma$. See text for further details.

a NS accretion efficiency of $\epsilon=e_{\text {acc }} k_{\text {def }}=0.30$. Accretion disk instabilities (e.g. van Paradijs 1996; Dubus et al. 2001; Coriat et al. 2012), which act to decrease the accretion efficiency in LMXBs, were not considered explicitly in this work, but are assumed to be integrated in the somewhat low accretion efficiency quoted above. Other mechanisms for inefficient accretion include propeller effects (Illarionov \& Sunyaev 1975) and direct irradiation of the donor's atmosphere by the pulsar (see Sect. 5.3 for further discussions). We calculated the Eddington mass-accretion rate using

$\dot{M}_{\text {Edd }}=2.3 \times 10^{-8} M_{\odot} \mathrm{yr}^{-1}\left(\frac{M_{\mathrm{NS}}}{M_{\odot}}\right)^{-1 / 3} \frac{2}{1+X}$,

where $X$ is the hydrogen mass fraction of the accreted material.

\section{Results}

\subsection{Parameters of the model grid}

In this work, we created a grid of models for LMXBs consisting of different initial donor star masses and NS masses, as well as for different values of the magnetic braking index, $\gamma$ (see Fig. 1). For each of these sets of parameters we tried a range of initial orbital periods, $P_{\text {orb }}$ in a systematic survey, yielding a total of roughly 400 models. More specifically, we chose the initial donor mass, $M_{2}$ between 1.1 and $1.6 M_{\odot}$ (all with a metallicity of $Z=0.02$ ). The lower mass limit is chosen to ensure nuclear evolution within a Hubble time. However, we find that $M_{2} \geq 1.2 M_{\odot}$ is often required for the stars to evolve through the LMXB phase and settle on the WD cooling track within a Hubble time. The upper mass limit is imposed by the requirement of a convective envelope on the ZAMS, which is an assumption needed to operate a magnetic wind. Although the 1.4-1.6 $M_{\odot}$ donors are borderline cases in this respect, we included them in our grid for comparison with previous studies in the literature. For the NSs, we applied initial masses of $M_{\mathrm{NS}}=1.2-1.9 M_{\odot}$. The initial $P_{\text {orb }}$ were mainly chosen in the range of 2 to 4 days and do not follow a uniform distribution. The reason for this is that we were interested in obtaining systems with certain properties which turned out to be located around a specific initial $P_{\text {orb }}$ which is characteristic for every studied configuration. To obtain this value 
A. G. Istrate et al.: Evolution of LMXBs below the bifurcation period

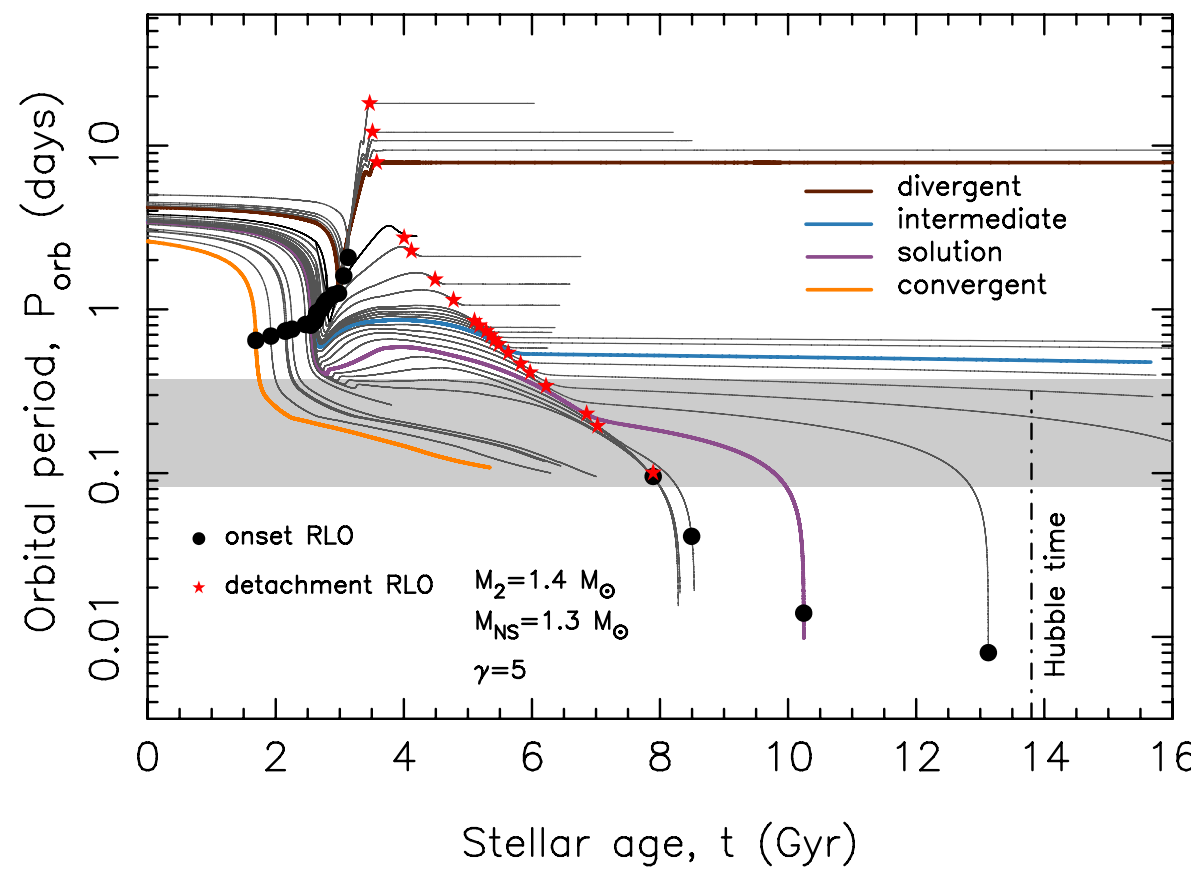

Fig. 2. Orbital period evolution for LMXB systems with a donor mass of $1.4 M_{\odot}$, a NS mass of $1.3 M_{\odot}$ and a magnetic braking index of $\gamma=5$. The black circles represent the onset of RLO and the red stars show the end of the mass-transfer phase. The observed MSP systems mainly investigated here, with low-mass He WD companions and $P_{\text {orb }}=2-9$ h (cf. Table 1), are located within the grey shaded region. The LMXB systems experience a second RLO and evolve into ultra-compact X-ray binaries in very tight orbits. we used a bracketing method which resulted in a high density of models around that specific initial $P_{\text {orb }}$. The magnetic braking index $\gamma$ was varied between 2 and 5 (but is constant for any given LMXB calculation). As mentioned in Sect. 3.2, in most cases we used a NS accretion efficiency parameter of $\epsilon=0.3$. However, for $1.5 M_{\odot}$ donors we also studied the influence of applying different values in the range $\epsilon=0.1-0.9$. Although this entire parameter space of variables is large, the resulting systems do show similarities in the evolution as discussed below.

\subsection{Orbital evolution, mass transfer and stellar structure}

The final $P_{\text {orb }}$ of a given LMXB is a result of the interplay between mass transfer, magnetic braking and gravitational wave radiation. It strongly depends on the initial $P_{\text {orb }}$ which determines the strength of orbital angular momentum losses and at which point in the nuclear evolution the donor star initiates RLO.

Pylyser \& Savonije (1988) classified the orbital evolution of an LMXB system with respect to the final $P_{\text {orb }}$ as: (i) converging, if $P_{\text {orb }}^{\text {final }}<P_{\text {orb }}^{\text {initial }}$; or (ii) diverging, if $P_{\text {orb }}^{\text {final }}>P_{\text {orb }}^{\text {initial }}$. Here, we redefine converging systems as those tight binaries where the donor star never detaches to form a He WD. As mentioned previously, we are interested in finding those systems that have a final $P_{\text {orb }}$ between $2-9 \mathrm{~h}$ and which have terminated their mass-transfer phase yielding a (proto) He WD remnant with a mass $<0.20 M_{\odot}(\mathrm{cf}$. Table 1$)$. The systems that fulfil these conditions are hereafter called solutions. Finally, we define the intermediate systems as those systems which detach from RLO to form a He WD with $9 \mathrm{~h}<P_{\text {orb }}^{\text {final }}<P_{\text {orb }}^{\text {initial }}$.

Figure 2 shows the variety in orbital period evolution for an initial configuration with $M_{2}=1.4 M_{\odot}(Z=0.02), M_{\mathrm{NS}}=$ $1.3 M_{\odot}, \gamma=5$ and $P_{\text {orb }}=2.6-5.0$ days. Highlighted with different colours are one example for each of the aforementioned classes of the outcome of LMXB evolution: converging, solution, intermediate and diverging. For clarity, we have omitted the markings of the temporary detachment of the diverging systems caused by the encounter of a slight chemical discontinuity at the outer boundary of the hydrogen burning shell (Tauris \& Savonije 1999; D’Antona et al. 2006). The converging systems never detach, but keep evolving towards the minimum orbital period, $P_{\min } \approx 10-85$ min (Paczynski \& Sienkiewicz 1981; Rappaport et al. 1982; Fedorova \& Ergma 1989; Ergma \& Sarna 1996; Podsiadlowski et al. 2002; van der Sluys et al. 2005a). As a result of numerical issues the evolution of the converging systems was ended before reaching $P_{\min }$. At this point they have masses $<0.13 M_{\odot}$ and yet a significant hydrogen content - even in their cores - and very small nuclear burning rates (cf. Figs. 4, 5, and 11). Hence, these systems will not detach and produce a He WD within several Hubble times (if ever).

As demonstrated in the literature (Tutukov et al. 1987; Pylyser \& Savonije 1988, 1989; Ergma \& Sarna 1996; Podsiadlowski et al. 2002; van der Sluys et al. 2005a; Ma \& Li 2009), an orbital bifurcation period $\left(P_{\text {bif }}\right)$ exists which separates the evolution of converging ${ }^{2}$ and diverging systems. With respect to the initial $P_{\text {orb }}$ on the ZAMS we find $P$ bif $\simeq 4.0$ days. As a result of magnetic braking the orbit shrinks during the mainsequence evolution of the donor star prior to mass transfer. Thus the bifurcation period at the onset of RLO is $P_{\text {bif }}^{\mathrm{RLO}} \simeq 1.2$ days (Fig. 2). If we apply other values of $M_{2}, M_{\mathrm{NS}}, \gamma$ or metallicity, the qualitative picture remains intact but the value of $P_{\text {bif }}^{Z A M S}$ changes between $\simeq 2.2-4.5$ days.

Figure 3 shows the RLO mass-transfer rate, $\left|\dot{M}_{2}\right|$ as a function of decreasing donor mass, $M_{2}$ for the four examples highlighted in Fig. 2. At first sight these rates are quite similar. From a closer look, however, it is seen that the wider systems have higher values of $\left|\dot{M}_{2}\right|$ (and shorter durations of RLO), as expected from an evolutionary point of view (e.g. Tauris \& Savonije 1999). And more importantly, the final fates of these four LMXB systems are quite different.

Changing the initial $P_{\text {orb }}$ between 2.6-4.5 days means that the systems will gradually shift from undergoing Case A (onset of RLO on the main sequence) to early Case B RLO (onset of RLO in the Hertzsprung-gap), as shown in Fig. 4. The converging systems start RLO relatively early when there is still a significant amount of hydrogen left in the core of the donor star. In these systems, the mass transfer is driven by a reduction

2 Here meaning converging, solutions and intermediate systems. 


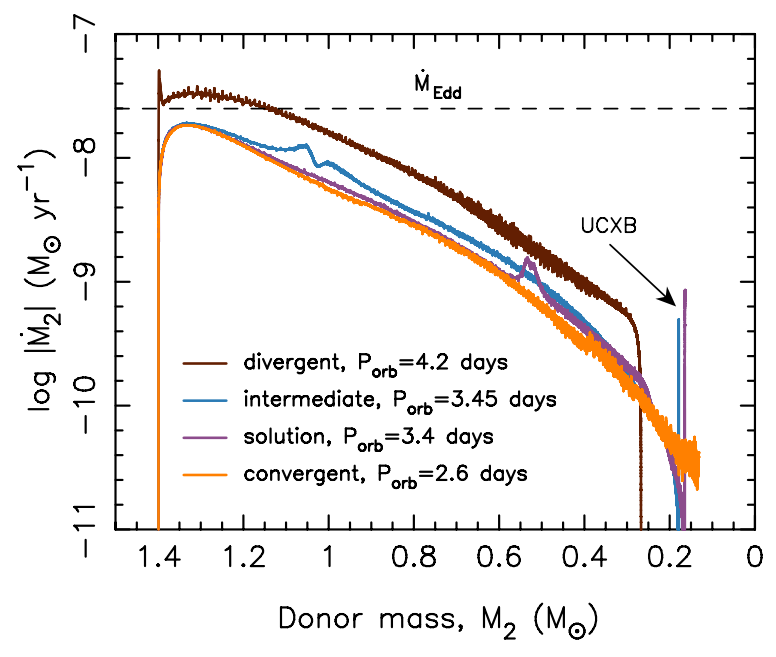

Fig. 3. Mass-transfer rate versus decreasing donor star mass for the four systems highlighted in Fig. 2. The larger the initial $P_{\text {orb }}$, the higher the mass-transfer rate and the shorter the RLO episode will be. The converging system does not detach at all. The system resulting in a solution eventually evolves into an ultra-compact X-ray binary (UCXB) when the He WD fills its Roche lobe.

in the orbital separation due to loss of orbital angular momentum (initially caused by magnetic braking, later dominated by gravitational wave radiation). The intermediate systems are in a transition between mass transfer driven by loss of orbital angular momentum and mass transfer driven by nuclear evolution and expansion of the donor star (the diverging systems).

The final helium abundance profiles of the donor stars are shown in Fig. 5. The thickness of the hydrogen-rich envelopes of these detached (proto) He WDs is very important for their subsequent thermal evolution (see Paper II).

In Fig. 6 we plot the final $P_{\text {orb }}$ ("final" refers to our last calculated model) versus the initial $P_{\text {orb }}$ for the same systems as in Fig. 2. The vertical dotted lines denote $P_{\text {detach }}, P_{\mathrm{UCXB}}$, and $P_{\text {bif }}$. We define $P_{\text {detach }}$ as the minimum initial $P_{\text {orb }}$ leading to a detached He WD companion, and $P_{\mathrm{UCXB}}$ as the maximum initial $P_{\text {orb }}$ leading to a system which becomes an ultra compact X-ray binary (UCXB) within a Hubble time (i.e. a detached system which, as a result of gravitational wave radiation, is driven into a very tight orbit with a second RLO from the He WD). All the systems on the left side of $P_{\text {detach }}$ are on their way to $P_{\min }$.

\subsubsection{The orbital period fine-tuning problem}

Figure 6 illustrates two important characteristics of our closeorbit LMXB modelling: (i) how sensitive the outcome is to the initial $P_{\text {orb }}$; (ii) the systems we refer to as solutions are produced within a very narrow interval of initial $P_{\text {orb }}=3.39-3.43$ days which corresponds to onset of RLO near $P_{\text {orb }} \approx 19.2-19.4 \mathrm{~h}$. The solutions produced in this study all start inside (or slightly beyond) the narrow interval of initial $P_{\text {orb }}$ between $P_{\text {detach }}$ and $P_{\mathrm{UCXB}}$. Those solutions with initial $P_{\mathrm{orb}}>P_{\mathrm{UCXB}}$ are the systems which, after detachment, do not evolve into UCXBs within a Hubble time. The width of the initial (ZAMS) range of $P_{\text {orb }}$ which allows for a solution is thus only $\sim 1 \%$ in $P_{\text {orb }}$.

This is a puzzling result given that a fair fraction of observed MSPs are found with He WDs and $P_{\text {orb }}=2-9 \mathrm{~h}$. We shall refer to this problem as the orbital period fine-tuning problem of LMXBs and discuss it further below, as well as in Sect. 5.2. Outside this narrow range in initial $P_{\text {orb }}$, the LMXB systems always evolve to become converging, intermediate or diverging systems.
In Fig. 7 we show the observed orbital period distribution of recycled pulsars with He WD companions (i.e. post-LMXB systems) in the Galactic field. Out of 35 systems with $P_{\text {orb }}<$ 10 days, 4 systems have $P_{\text {orb }}<9 \mathrm{~h}$. Assuming these 4 systems (resembling the solutions from our modelling) are indeed produced from LMXBs with an initial $P_{\text {orb }}$ between 3.39-3.43 days (i.e. corresponding to the lower $\sim 4 \%$ of the full interval of initial $P_{\text {orb }}$, roughly between 3.4-4.4 days, which lead to formation of MSPs with He WDs and final $P_{\text {orb }}<10$ days, see Fig. 6), we can estimate the probability for this outcome being a chance coincidence. In that case, the other 31 systems are produced for initial $P_{\text {orb }}=3.43-4.4$ days $\left(~ 96 \%\right.$ of the interval of initial $P_{\text {orb }}$ producing MSPs with final $P_{\text {orb }}<10$ days). If the pre-LMXB distribution of $P_{\text {orb }}$, following the SN explosion that created the NS, is approximately flat between $\sim 3.4-4.4$ days, then the probability for producing at least 4 out of 35 MSPs with $P_{\text {orb }}<9$ h is about $1: 20$, i.e. corresponding to not being a chance coincidence at the $95 \%$ confidence level.

We calculated this probability analytically using the binomial cumulative distribution function.

Actually, the problem is even worse given that a certain fraction of the systems formed with $P_{\text {orb }} \simeq 2-9$ h will merge because of gravitational wave radiation and thus not be observable for as long time as the MSPs with larger $P_{\text {orb }}$. (The expected radio lifetimes of MSPs are many Gyr and are independent of $P_{\text {orb }}$.) Hence, more than 4 systems are most likely to have formed as solutions for every 31 systems produced with $P_{\text {orb }}$ between $9 \mathrm{~h}$ and 10 days. As an example, PSR J0348+0432 ( $P_{\text {orb }}=$ $2.46 \mathrm{~h}$, cf. Table 1) has a merger timescale of only $\sim 400 \mathrm{Myr}$ (Antoniadis et al. 2013). Additionally, there are observational selection effects against finding accelerated pulsed signals in very close binaries (Johnston \& Kulkarni 1991), although modern day acceleration search software and increased computer power have somewhat alleviated this problem.

The discrepancy between observational data and our calculations is further illustrated by the red dashed line in Fig. 7 which shows the rough distribution of systems expected from modelling with standard input physics (assuming again a flat distribution of initial $P_{\text {orb }}$ between $~ 3.4-4.4$ days.)

The statistics depends, of course, on how the exact subsamples are chosen. However, the above example was calculated for the most conservative case using the result of calculated models with $\gamma=5\left(M_{2}=1.4 M_{\odot}\right)$. As we demonstrate below, the required fine-tuning is much worse (>99.99\% C.L.) for smaller values of $\gamma$. Therefore, there is no doubt that this severe finetuning has its basis in the input physics currently adopted in standard LMXB modelling. Something seems to be missing or must be modified - some mechanism that funnels more LMXBs to end up as MSPs with detached He WDs and $P_{\text {orb }}<9 \mathrm{~h}$.

\subsection{Magnetic braking and the influence of the $\gamma$-index}

As discussed previously, the magnetic braking law is not well known. For this reason we investigated how the general behaviour of LMXBs in close orbits changes with different values of $\gamma$. In Fig. 8 is shown the difference in orbital period evolution for an initial LMXB with $M_{2}=1.4 M_{\odot}, M_{\mathrm{NS}}=1.3 M_{\odot}$ and $P_{\text {orb }}=2.8$ days, for four different values of $\gamma: 2,3,4$ and 5. One can see that the higher the $\gamma$-index, the stronger is the loss of orbital angular momentum due to magnetic braking. This situation is reversed once the donor radius decreases below $1 R_{\odot}$ (cf. Eq. (5)). However, at that point the orbital evolution is mainly dominated by mass loss. 
A. G. Istrate et al.: Evolution of LMXBs below the bifurcation period
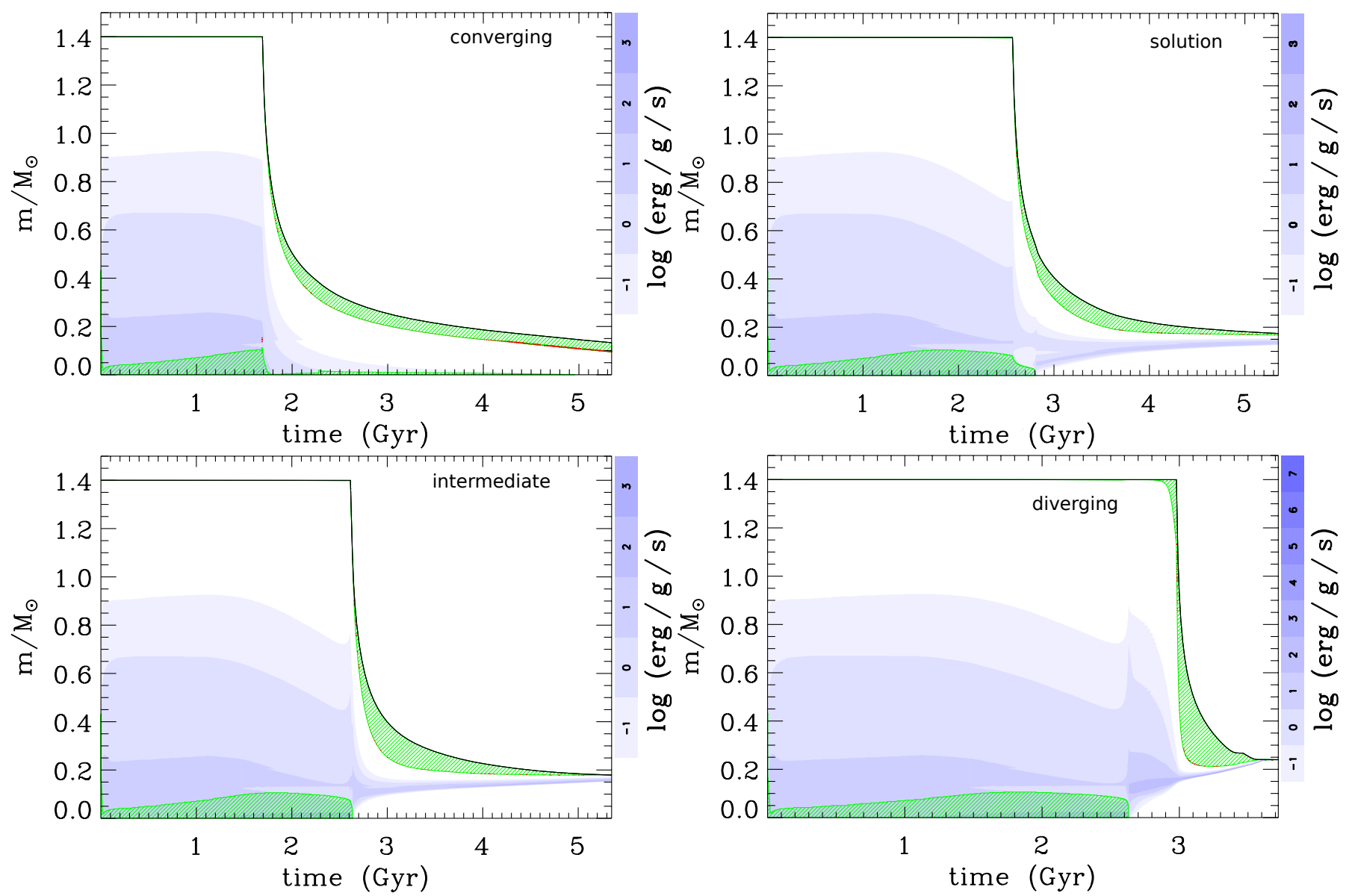

Fig. 4. The Kippenhahn diagram of a converging (top left), solution (top right), intermediate (bottom left) and diverging (bottom right) LMXB system, respectively. In all cases we used $M_{2}=1.4 M_{\odot}, M_{\mathrm{NS}}=1.3 M_{\mathrm{NS}}, \gamma=5$ and applied initial $P_{\text {orb }}=2.6,3.4,3.45$ and 4.2 days, respectively. These four systems are identical to the examples highlighted in Fig. 2. In the converging system the donor star experiences Case A RLO; for the solution and intermediate systems the mass transfer is either late Case A or early Case B RLO, while the diverging systems undergo Case B RLO. The plots show cross-sections of the stars in mass-coordinates from the centre to the surface of the star, along the $y$-axis, as a function of stellar age on the $x$-axis. For clarity, we only show the evolution up to a stellar age of $5.35 \mathrm{Gyr}$ in the first three panels. The duration of the LMXBphase is: " $\infty$ " (no detachment), $4.0 \mathrm{Gyr}, 2.9 \mathrm{Gyr}$ and $0.6 \mathrm{Gyr}$, respectively. The green hatched areas denote zones with convection (according to the Schwarzschild criterion), initially in the core and later in the envelope of the donor stars. The intensity of the blue color indicates the net energy-production rate; the hydrogen burning shell is clearly seen in the case of the solution, intermediate and diverging systems at $m / M_{\odot} \simeq 0.2$.

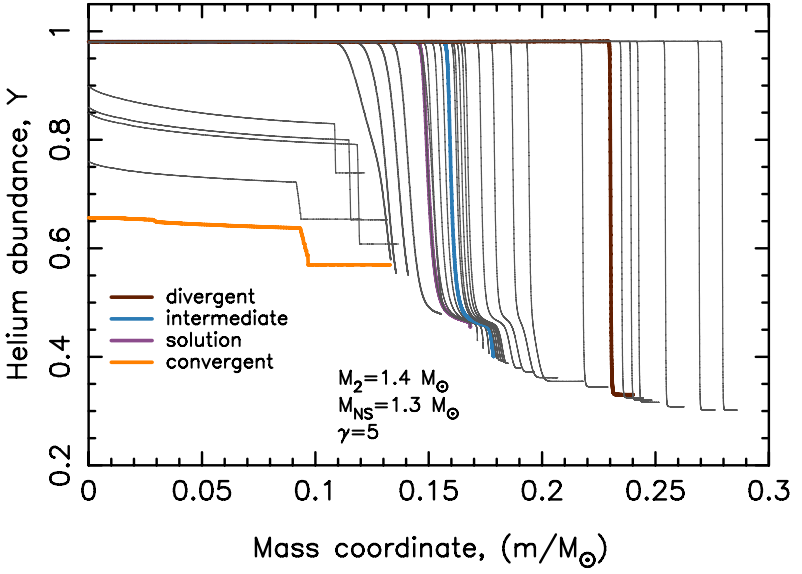

Fig. 5. Helium abundance profiles of the donor stars in Fig. 2 at the time of RLO detachment (diverging, intermediate and solution) or at the end of our calculations (converging).

The main consequence of varying the $\gamma$-index is that $P_{\text {detach }}$, $P_{\mathrm{UCXB}}$ and $P_{\text {bif }}$ have smaller values for smaller $\gamma$, but the general orbital behaviour is similar. However, the final fate of the
LMXBs is seen to be quite a sensitive function of $\gamma$, and the orbital period fine-tuning problem gets worse for $\gamma<5$. For example, in Fig. 9 we demonstrate that for $\gamma=2$ the resulting width of the interval of initial $P_{\text {orb }}$ leading to an observed solution is less than $2 \min \left(<0.05 \%\right.$ in $\left.P_{\text {orb }}\right)$. This translates the significance of the orbital period fine-tuning problem to $>99.99 \%$ C.L. In Fig. 10 we have demonstrated how the orbital period fine-tuning problem systematically exacerbates with lower values of $\gamma$.

In the literature, some authors (e.g. Podsiadlowski et al. 2002; van der Sluys et al. 2005a) reduced the magnetic braking by an ad-hoc factor related to the size of the convective envelope, or turned it off when the donor became fully convective. We note here that with our code we did not produce any donors that became fully convective. In Sect. 5.1 we discuss the magnetic braking law and compare our results with previous work in the literature.

\subsection{Evolution in the HR-diagram and hydrogen shell flashes}

Figure 11 shows the evolution in the Hertzsprung-Russell diagram of the donor stars for all the systems in Fig. 2. The systems that start mass transfer during hydrogen core burning (converging Case A RLO systems) closely follow an evolution studied in 


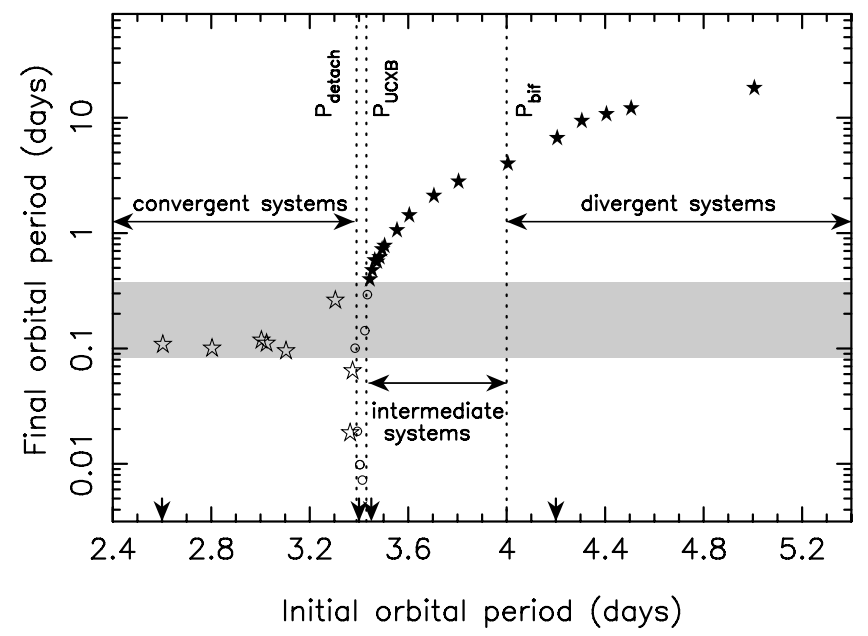

Fig. 6. Final $P_{\text {orb }}$ versus initial $P_{\text {orb }}$ for all binaries investigated with $M_{2}=1.4 M_{\odot}, M_{\mathrm{NS}}=1.3 M_{\odot}$ and a magnetic braking index of $\gamma=5$ (cf. Fig. 2). The arrows at the bottom indicate the initial $P_{\text {orb }}$ of the four highlighted systems. Circles represent solutions, i.e. systems which detached while situated inside the grey shaded region that marks the location of the observed MSP systems with $P_{\text {orb }}=2-9$ h. The values of initial $P_{\text {orb }}$ for these systems are confined to an extremely narrow interval (the orbital period fine-tuning problem, cf. Sect. 4.2.1). Open stars represent converging systems (which do not detach) and for which we ended our evolutionary calculations before reaching $P_{\min }$. The vertical dotted lines represent $P_{\text {detach }}, P_{\mathrm{UCXB}}$, and $P_{\text {bif }}-$ see text.

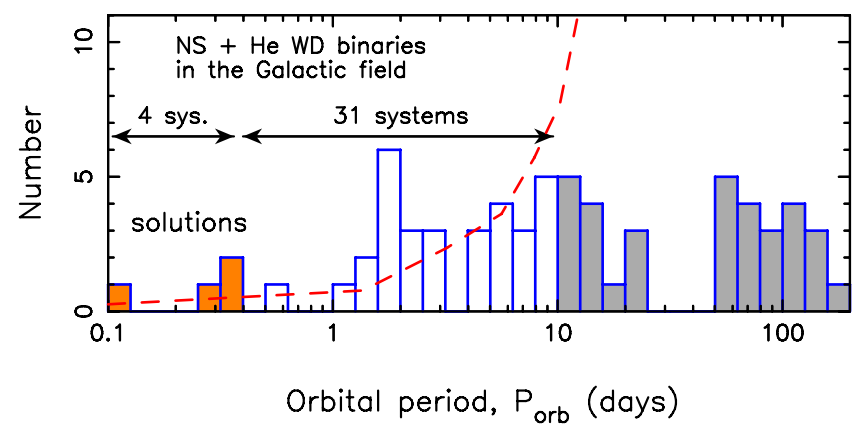

Fig. 7. Distribution of orbital periods for all observed recycled pulsars (MSPs) with He WD companions in the Galactic field. In total there are 35 systems with $P_{\text {orb }}<10$ days; four of these are the solutions $\left(P_{\text {orb }}<9\right.$ h) marked with orange. Data from the ATNF Pulsar Catalogue Manchester et al. (2005) - version 1.50, July 2014. The red dashed line shows the distribution of systems that would be expected from our calculations using standard input physics (i.e. using the results presented in Fig. 6), thus illustrating the orbital period fine-tuning problem.

detail by Pylyser \& Savonije (1989). These stars evolve along an almost straight line (following the ZAMS, with smaller radii as they lose mass) down towards very low temperatures and very low luminosities, until the donor star almost becomes fully convective and approaches the Hayashi-track. Subsequently, the luminosity is seen to decrease relatively rapidly because of fading nuclear burning and increasing degeneracy. The systems that fill their Roche lobe close to the core contraction phase or later, such as the solutions, the intermediate and the diverging systems, first evolve towards low effective temperatures and low luminosities until, because of ignition of hydrogen shell burning, they turn towards higher temperatures and - for the diverging systems with (sub)giant donors - higher luminosities. The more evolved the donor star is towards the red-giant branch (RGB) when it initiates mass transfer (i.e. the more massive its core mass), the

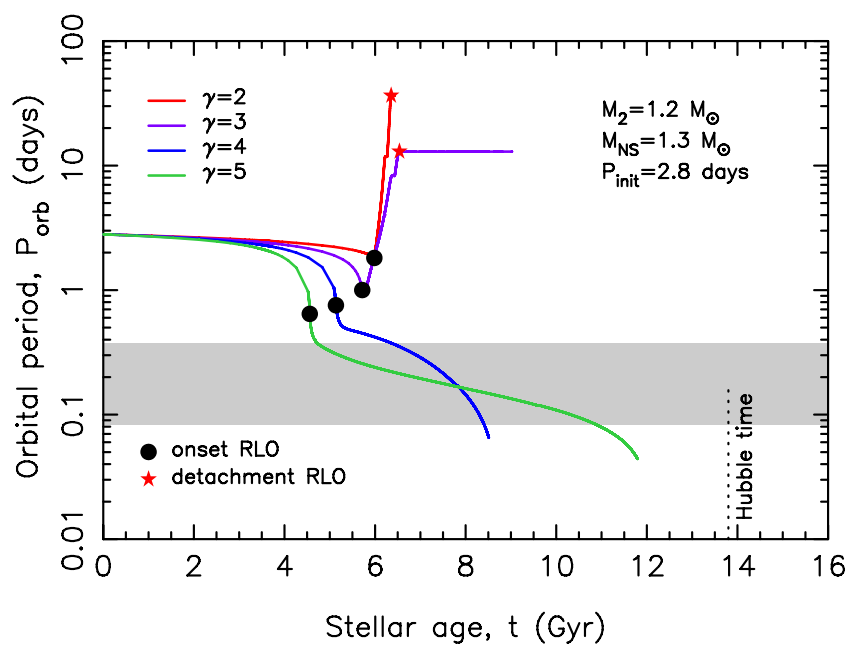

Fig. 8. Influence of the magnetic braking index, $\gamma$ on the orbital evolution of an LMXB system with $M_{2}=1.2 M_{\odot}, M_{\mathrm{NS}}=1.3 M_{\odot}$ and initial $P_{\text {orb }}=2.8$ days. Plotted is $P_{\text {orb }}$ as a function of age of the donor star. None of these models produce a solution (i.e. detachment within the grey shaded region). Instead the outcome is two diverging and two converging systems.

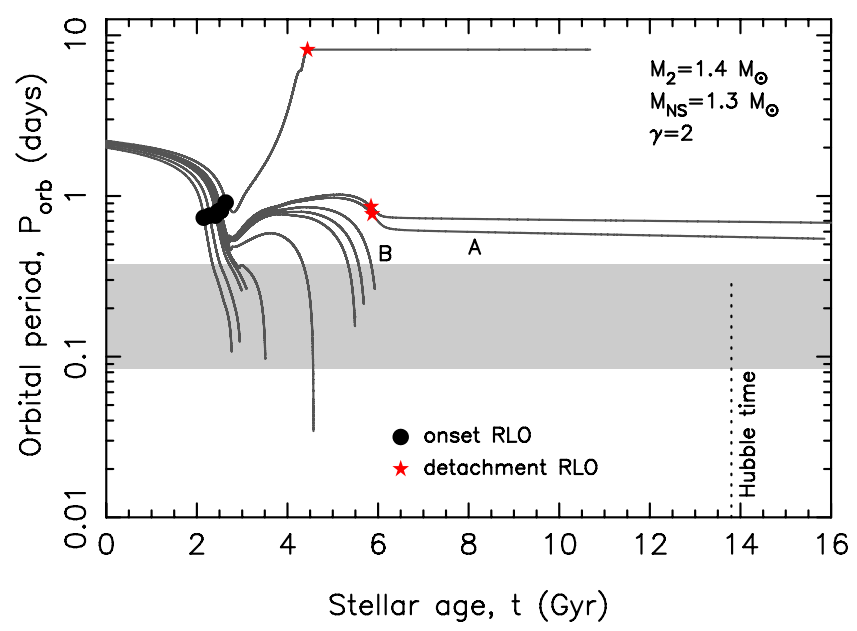

Fig. 9. Orbital period evolution for all LMXB systems investigated with $M_{2}=1.4 M_{\odot}, M_{\mathrm{NS}}=1.3 M_{\odot}$ and a magnetic braking index of $\gamma=2$. The first detached system (A) has an initial $P_{\text {orb }}=2.148$ days while the widest of the converging systems investigated (B) has an initial $P_{\text {orb }}=2.147$ days. Whereas system A leads to an intermediate system (with a final $P_{\text {orb }}>9$ h), system B does not detach but keeps evolving towards $P_{\min }$. Therefore, the observed solutions (i.e. RLO detachment and formation of an MSP and a He WD with $P_{\text {orb }}=2-9$ h) would require a fine-tuning of the initial $P_{\text {orb }}$ to be in a narrow range of less than 2 min. For larger values of $\gamma$ the situation is less severe (Fig. 2) but still a serious problem, see Sects. 4.2.1, 4.3, and 5.2.

higher the luminosity will be when the star finally evolves towards the WD cooling track.

To get a better overview of the correspondence between the evolutionary status of the donor star at the onset of RLO and the final fate of the LMXB, we show in Fig. 12 a zoom-in along the evolutionary track in the HR-diagram of a $1.4 M_{\odot}$ star, from the ZAMS to the point where mass transfer is initiated to a 1.3 $M_{\odot}$ NS companion with various values of initial $P_{\text {orb }}$. The marked points indicate a sequence of cases where the evolutionary status of the donor star at the onset of RLO is somewhere between the middle of the main sequence (A) and all the way 


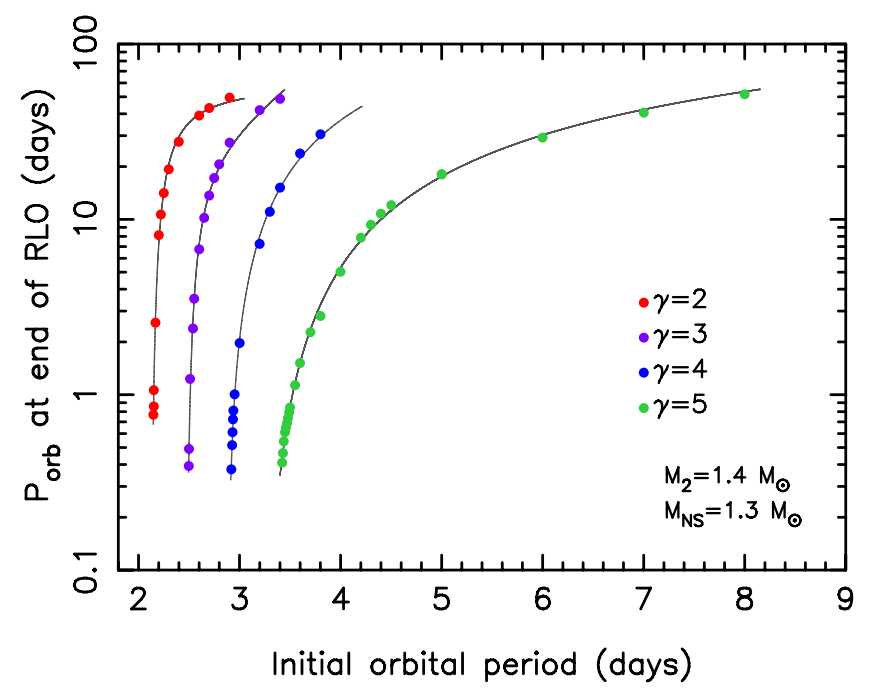

Fig. 10. Calculated $P_{\text {orb }}$ at Roche-lobe detachment as a function of initial $P_{\text {orb}}$, plotted for different values of the magnetic braking index, $\gamma$. It is seen how the orbital period fine-tuning problem becomes worse for smaller values of $\gamma$, see text for discussions.

up to the point $(\mathrm{F})$ where the donor star has ascended on the RGB. The systems leading to solutions initiate mass transfer in a very narrow epoch between points $\mathrm{B}$ and $\mathrm{C}$, when the donor star leaves the main sequence and starts the contraction phase. The converging systems initiate mass transfer between points A and $\mathrm{B}$, while the intermediate and diverging systems start mass transfer after points $\mathrm{C}$ and $\mathrm{D}$, respectively.

One can see from Fig. 11 that there is a region in which the donor stars experience one or several hydrogen shell flashes. The intensity of the flashes is gradually increasing with the initial $P_{\text {orb }}$. In the literature, the mass interval in which a proto-WD experiences hydrogen flashes is roughly between $0.2-0.4 M_{\odot}$ (Driebe et al. 1998; Sarna et al. 2000; Althaus et al. 2001, 2013; Serenelli et al. 2002; Nelson et al. 2004; Panei et al. 2007), depending on metallicity and input physics (primarily in treatment of diffusion). Donor stars which initiate RLO in the interval between points $\mathrm{E}$ and $\mathrm{F}$ in Fig. 12 will experience hydrogen shell flashes after the mass-transfer phase. Some of these flashes may cause additional RLO of small amounts of material $\left(\sim 5 \times 10^{-4} M_{\odot}\right)$. A more complete discussion on the observed flashes in our models, and thermal evolution of (proto) WDs in particular, can be found in Paper II.

\subsection{The ( $\left.M_{\mathrm{WD}}, P_{\mathrm{orb}}\right)$-relation for tight orbits}

For low-mass stars $\left(<2.3 M_{\odot}\right)$ on the RGB, there is a well-known relationship between the mass of the degenerate helium core and the radius of the giant star - almost entirely independent of the mass present in the hydrogen-rich envelope (Refsdal \& Weigert 1971; Webbink et al. 1983). This relationship is very important for the formation of binary MSPs because it results in a unique relation between their orbital period $\left(P_{\mathrm{orb}}\right)$ and WD mass $\left(M_{\mathrm{WD}}\right)$ following the LMXB mass-transfer phase (Savonije 1987; Joss et al. 1987; Rappaport et al. 1995; Tauris \& Savonije 1999; Nelson et al. 2004; De Vito \& Benvenuto 2010; Shao \& Li 2012). The masses of the He WD companions are expected to be between $0.13<M_{\mathrm{WD}} / M_{\odot}<0.46$. The predicted correlation between $M_{\mathrm{WD}}$ and $P_{\text {orb }}$ has previously been somewhat difficult to verify observationally since few MSPs had accurately measured masses of their companion star. However, over the past decade the correlation has been confirmed from mass measurements obtained from e.g. pulsar timing (Shapiro delay) or optical observations of He WD companions (e.g. van Kerkwijk et al. 2005), see Tauris \& van den Heuvel (2014) for a recent comparison of theory and data.

As a consequence of loss of orbital angular momentum due to magnetic braking, LMXB systems with initial $P_{\text {orb }} \lesssim P_{\text {bif }}$ are expected to end up as close-binary MSPs with $P_{\text {orb }}$ as short as a few hours (Sect. 3.1.3). Therefore, because of the still unknown strength of magnetic braking, the $\left(M_{\mathrm{WD}}, P_{\mathrm{orb}}\right)$-relation has always been considered less trustworthy for binary pulsars with $P_{\text {orb }} \lesssim 2$ days (where He WDs have masses $<0.20 M_{\odot}$ ).

In Fig. 13 we have plotted the final $P_{\text {orb }}$ versus $M_{\mathrm{WD}}$ for all our detached systems. For comparison we have plotted the theoretical $\left(M_{\mathrm{WD}}, P_{\mathrm{orb}}\right)$-relation following Tauris \& Savonije (1999), hereafter TS99. Whereas this relation is expected out to $P_{\text {orb }} \simeq 1000$ days for He WDs (at which point the core mass exceeds $\sim 0.46 M_{\odot}$ and He is ignited, leading to a continuation of the correlation for higher mass CO WDs produced in LMXBs), Fig. 13 only shows the lower left part of the full diagram. The analytical expression of TS99 was derived for $P_{\text {orb }} \gtrsim 1$ day. However, as noted, for example, by van Kerkwijk et al. (2005), Bassa et al. (2006a), Antoniadis et al. (2012) and Corongiu et al. (2012), even for $P_{\text {orb }}<1$ day there is apparently a fairly good agreement between the measured masses of He WDs and those expected from the theoretical $\left(M_{\mathrm{WD}}, P_{\mathrm{orb}}\right)$-relation. Examples of observational data include: PSR J1738+0333 (Antoniadis et al. 2012), PSR J1910-5959A (Corongiu et al. 2012), PSR J1012+5307 (Lazaridis et al. 2009), PSR J0348+0432 (Antoniadis et al. 2013), PSR J0751+1807 (Nice et al. 2008) ${ }^{3}$; as well as low-mass He WD companions to non-degenerate stars (e.g. van Kerkwijk et al. 2010; Breton et al. 2012; Maxted et al. 2014).

At first sight, PSR J0348+0432 may seem to be have an observed $P_{\text {orb }}(2.46 \mathrm{~h})$ a bit below the expected theoretical value. However, one must keep in mind the effect of gravitational wave radiation following the detachment of the binary. Antoniadis et al. (2013) estimated a cooling age for this WD of about 2 Gyr, meaning that $P_{\text {orb }} \simeq 5 \mathrm{~h}$ at the moment of Roche lobe detachment (a factor two larger than its present value). See also Fig. 2 for the effect of gravitational wave radiation from the detached binaries (solutions). In addition, low metallicity stars have smaller radii which leads to smaller values of final $P_{\text {orb }}$ for the He WDs (see also Jia \& Li 2014, for a recent investigation of this effect in close-orbit systems).

To summarize, for final $P_{\text {orb }}$ less than a few days, our LMXB modelling demonstrates, as expected, a significant spread in the distribution of systems with respect to an extension of the relation of TS99. The deviations seem to be semi-systematic, in the sense that all models have smaller values of $M_{\mathrm{WD}}$ compared to the extrapolation of TS99, and there is a clear division of tracks depending on the original mass of the donor star, $M_{2}$. However, interestingly enough the order of these tracks does not follow a monotonic change in $M_{2}$. Nevertheless, the modelling of the correlation between $M_{\mathrm{WD}}$ and $P_{\mathrm{orb}}$ remains surprisingly robust for tight orbits, albeit with larger scatter. Given this large scatter (for example, we find that $P_{\text {orb }}$ can vary by a factor of four for $M_{\mathrm{WD}} \simeq 0.16 M_{\odot}$, cf. Fig. 13) it is somewhat meaningless to provide an exact analytical fit for $P_{\text {orb }}<2$ days. In this study, we only modelled systems with a metallicity $Z=0.02$. Accounting

A more recent He WD mass constraint for PSR J0751+1807 is $M_{\mathrm{WD}}=0.138 \pm 0.006 M_{\odot}(95 \%$ confidence level, Nice, priv. comm. . This value is used in Fig. 13 and Table 1. 


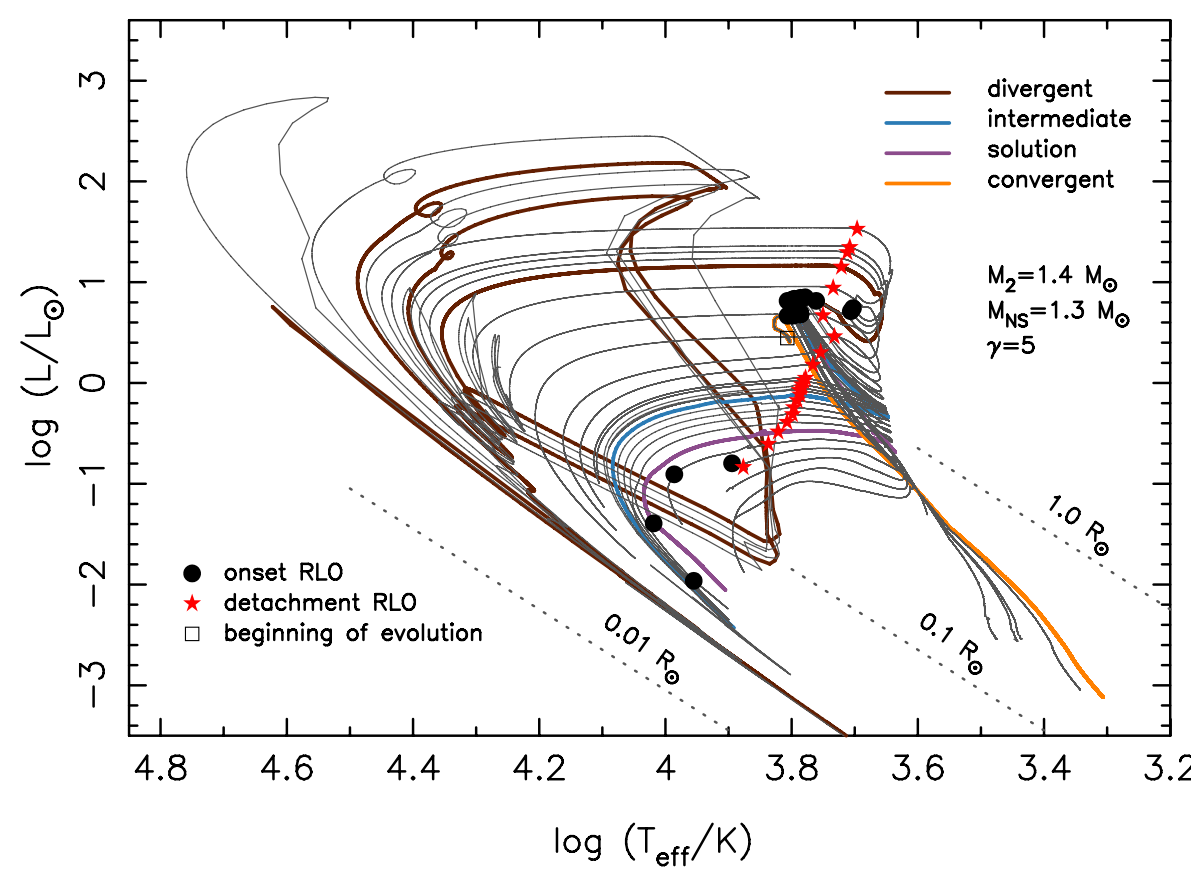

Fig. 11. Evolutionary tracks in the HR-diagram for the systems shown in Fig. 2. The small square on the ZAMS near $\left(\log T_{\text {eff }}, \log \left(L / L_{\odot}\right)\right)=$ $(3.8,0.5)$ marks the beginning of the evolution for all systems.

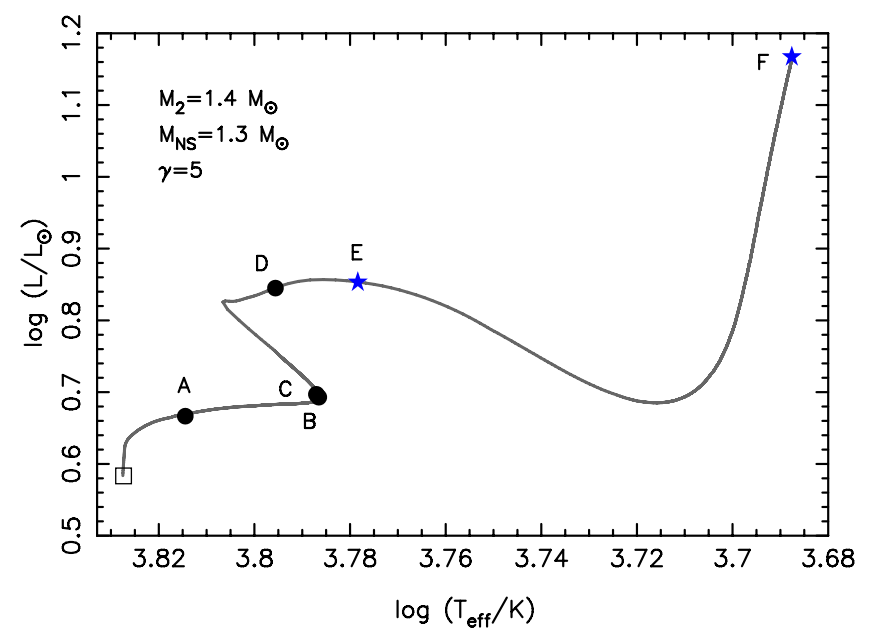

Fig. 12. HR-diagram for the evolution of a $1.4 M_{\odot}$ star $(Z=0.02)$. Along the evolutionary track (starting from the ZAMS marked by a square) is shown the points where such a donor star would initiate RLO to a $1.3 M_{\odot}$ NS. The black circles represent the beginning of mass transfer for a system with initial $P_{\text {orb }}$ of 2.6 (A), 3.38 (B), 3.42 (C), 3.6 (D), $4.0(\mathrm{E})$ and 7.0 days $(\mathrm{F})$, respectively. The interval between the points $\mathrm{E}$ and $\mathrm{F}$ denote an epoch where the donor star experiences hydrogen shell flashes after the end of the mass-transfer phase.

for stars with other metallicities (e.g. TS99; Jia \& Li 2014), we therefore expect a broader scatter of He WD masses between 0.14-0.20 $M_{\odot}$ for the systems with $P_{\text {orb }}<2$ days.

\subsubsection{Minimum mass of a He WD}

From theoretical work, it is expected that degenerate He WDs have masses of at least $0.13 M_{\odot}$ (Schönberg \& Chandrasekhar 1942; Tutukov et al. 1987). Indeed, we find that all our calculated He WDs have masses $M_{\mathrm{WD}} \geq 0.15 M_{\odot}$. Donor stars in converging LMXBs with smaller semi-degenerate cores have relatively thick hydrogen-rich envelopes. Therefore these stars remain hydrogen rich and bloated which prevents them from terminating their mass-transfer process and forming a detached He WD. Such donors, which often suffer from ablation via the pulsar wind, can have their masses reduced significantly, leading to black-widow type eclipsing MSP systems which have typical companion masses of a few $0.01 M_{\odot}$ (Roberts 2013; Chen et al. 2013), or even complete evaporation and formation of an isolated MSP; in some cases possibly surrounded by an asteroid belt (Shannon et al. 2013).

\section{Discussion}

\subsection{The magnetic braking law}

The loss of orbital angular momentum by magnetic braking is an uncertain aspect of LMXB evolution in close systems. For many years it has been thought that the magnetic field has to be anchored in underlying radiative layers of a star (Parker 1955). However, more recent observations and theoretical calculations question this hypothesis (e.g. Dorch \& Nordlund 2001; Barnes 2003; Barnes \& Kim 2010; Hussain 2011), and suggest that even fully convective stars may still operate a significant magnetic field - a conclusion which also has important consequences for the explanation of the observed period gap in CVs (Spruit \& Ritter 1983; Knigge et al. 2011).

In addition, it is possible that the stellar activity necessary for magnetic braking to operate may saturate for rotation periods shorter than a few days (Rucinski 1983; Vilhu \& Walter 1987). This would lead to a much flatter dependence of the angular momentum loss rate on the angular velocity $\left(\dot{J}_{\mathrm{MB}} \propto \Omega^{1.2}\right)$ than is given by the Skumanich-law ( $\dot{J}_{\mathrm{MB}} \propto \Omega^{3}$, Skumanich 1972) on which basis Eq. (5) is derived (see also Verbunt \& Zwaan 1981). Based partly on observational work, Stepien (1995) derived a new magnetic braking law which smoothly matches the Skumanich-law for wide systems to the dependence obtained by Rucinski (1983) for short orbital period systems ( $\$ 3$ days):

$\frac{\dot{J}_{\mathrm{MB}}}{J_{\text {orb }}} \simeq-1.90 \times 10^{-16} \frac{k^{2} R_{2}^{2}}{a^{2}} \frac{M^{2}}{M_{1} M_{2}} \mathrm{e}^{-1.50 \times 10^{-5} / \Omega} \quad \mathrm{s}^{-1}$. 


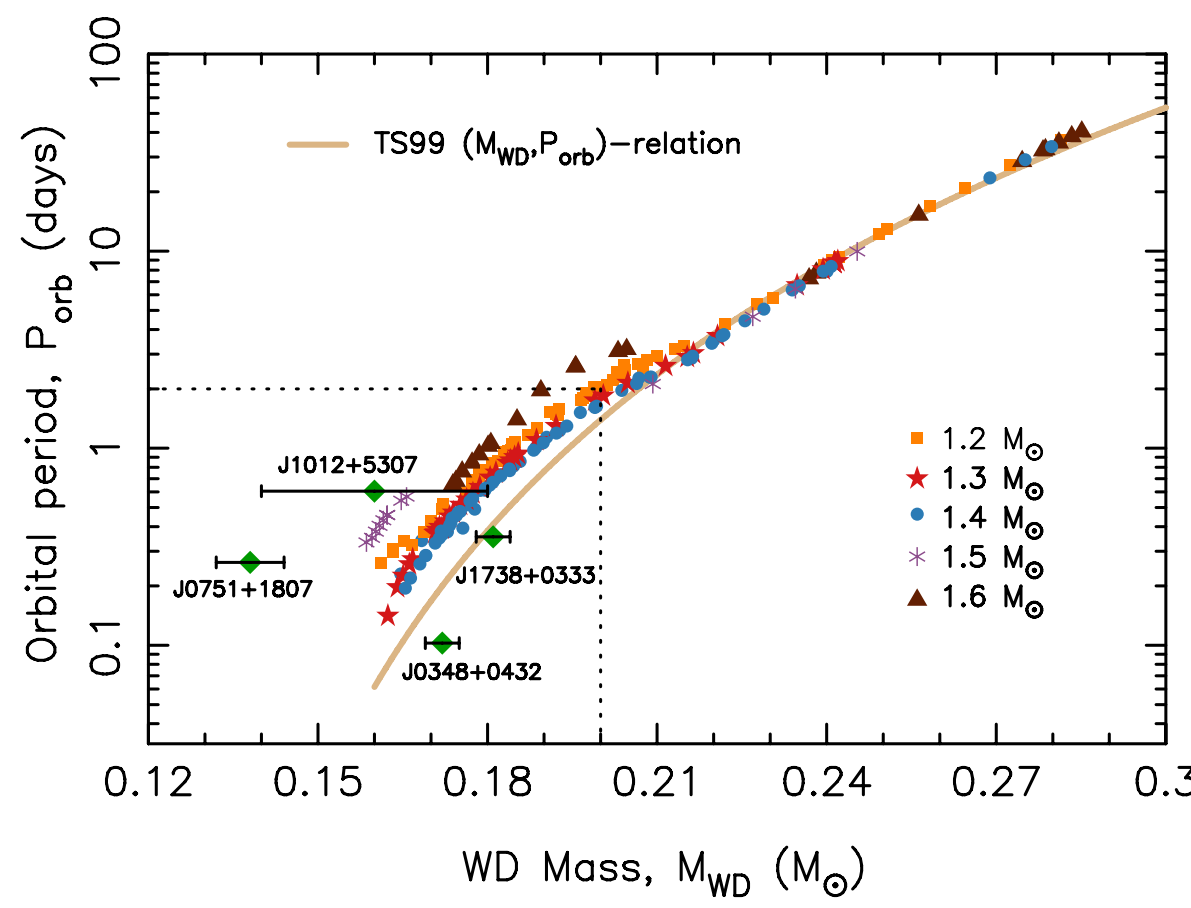

Fig. 13. $\left(M_{\mathrm{WD}}, P_{\mathrm{orb}}\right)$-diagram for all the studied LMXB systems which produced a detached $\mathrm{He}$ WD. The initial parameters were chosen from: $M_{2}=1.2-1.6 \quad M_{\odot}, M_{\mathrm{NS}}=$ 1.3-1.9 $M_{\odot}, \gamma=2-5$, and $\epsilon=0.1-0.9$. The thick curve (TS99) represents the analytical expression given in Tauris \& Savonije (1999) for $Z=0.02$ (which was derived only for $P_{\text {orb }} \gtrsim$ 1 day, but here for illustration extended down to smaller values of $P_{\text {orb }}$ ). The green diamonds represent observed MSP systems with He WDs and $P_{\text {orb }}<15$ h (i.e. similar to the solutions and partly the intermediate systems of our modelling). The region where the analytical expression is uncertain is for $P_{\text {orb }}<2$ days (and $\left.M_{\mathrm{WD}} \lesssim 0.20 \quad M_{\odot}\right)$. Although our calculations show a larger spread of the systems in this region the modelling of this correlation is still surprisingly robust - see text.
Equation (5) and the formula above represent a strong and a weak magnetic braking torque, respectively, and their relative strength can be compared in e.g. Tauris (2001). For detailed investigations and reviews on the magnetic wind and the braking torque, see e.g. Eggleton (2001), van der Sluys et al. (2005b) and Knigge et al. (2011), and references therein. In our work presented here, we have restricted ourselves to allow for a variation in the magnetic braking strength by varying the $\gamma$-index in Eq. (5). This was partially motivated by the results of the work by van der Sluys et al. (see below) who applied Eq. (8) without success.

\subsection{Further evidence of an orbital period fine-tuning problem}

As demonstrated so far in this paper, we have a problem with modelling the formation of MSPs with He WDs in tight orbits. From a closer look in the literature it is evident that there is independent support for this conclusion, and our numerical studies are no exception from a more general picture.

van der Sluys et al. (2005a,b) investigated the evolution of LMXBs with the aim of producing UCXBs within a Hubble time. Using detailed modelling of LMXBs they concluded in their first paper that only a narrow range of initial $P_{\text {orb }}$ and $M_{2}$ is able to result in parameters similar to those of observed UCXBs. To solve this problem, in their second paper, they applied reduced magnetic braking to their models following the work of Sills et al. (2000). The outcome was, however, that for less efficient magnetic braking it becomes impossible to evolve any systems to UCXBs.

In addition, we can compare our results with the detailed studies by Podsiadlowski et al. (2002) and Lin et al. (2011). In the work by Podsiadlowski et al. (2002), no solutions are found in their Fig. 13. Only sequence $d$ in their Fig. 16 leads to a solution. In Fig. 5 of Lin et al. (2011), one can see that only a few systems, out of $\sim 14000$ pulsar-WD binaries, are produced with a detached low-mass He WD orbiting a pulsar with $P_{\text {orb }}<15$ h. The orbital period fine-tuning problem is also seen indirectly in Fig. 6 (right panel) of Jia \& Li (2014) where a small relative change in the initial $P_{\text {orb }}$ results in a large relative change in the final $M_{\mathrm{WD}}$, i.e. $\Delta M_{\mathrm{WD}} / M_{\mathrm{WD}}>30 \Delta P_{\mathrm{orb}} / P_{\text {orb }}$.
A related problem is the question of truncating the RLO. In their analysis of the formation of PSR J0348+0432, Antoniadis et al. (2013) concluded that its existence requires a finely tuned truncation of the mass-transfer process which is not yet understood. (This system is investigated in our Paper III.)

It seems clear that there is evidence of a general problem of reproducing tight-orbit pulsar binaries using current stellar evolution codes. The converging LMXBs most often do not detach but keep evolving with continuous mass transfer to more and more compact systems with $P_{\text {orb }} \leq 2 \mathrm{~h}$ and ultra-light donor masses $M_{2}<0.08 \mathrm{M}_{\odot}$. In the few instances where fine-tuning may lead to detachment at the right values of $P_{\text {orb }}$ and $M_{2}$, the donor star is typically too hydrogen rich to settle and cool as a compact He WD. Instead the evolution may lead to formation of a redback-like system (Roberts 2013; Chen et al. 2013) which switches back and forth between being visible as an X-ray binary and an eclipsed radio MSP with a bloated companion (e.g. Archibald et al. 2009; Papitto et al. 2013; Bassa et al. 2014).

All of the above-mentioned modelling of LMXBs has difficulties producing detached He WDs with $P_{\text {orb }}=2-9$ h (referred to in this paper as solutions). As demonstrated in Sect. 4.2.1, this is in clear contradiction with observations which show a relatively large population of such systems. Although we were able to produce solutions for all choices of $M_{2}=1.2-1.6 M_{\odot}$ and values of $\gamma=2-5$, it seems to require an unrealistic high degree of fine-tuning. Hence, we conclude that apparently something is missing in the standard input physics applied for LMXB modelling.

\subsection{Irradiation effects, accretion disk instabilities and circumbinary disks}

Several effects may potentially affect the LMXB evolution, such as irradiation of the donor star, accretion disk instabilities and a circumbinary disk. As discussed below, we have neglected these effects in our work presented here. Firstly, because in this study we want to isolate the investigation of magnetic braking. Secondly, it has been demonstrated that these effects are uncertain and difficult to quantify for trustworthy modelling. We now briefly discuss each of these effects. 
During the LMXB evolution, a small part of mass lost from the companion may be injected into a circumbinary disk, which will exert tidal torques on the binary and extract angular momentum from the system (van den Heuvel 1994; Spruit \& Taam 2001). In addition, feedback mechanisms caused by tides may transfer angular momentum from the disk back into the binary (Lin \& Papaloizou 1979). Whether or not such a circumbinary disk may act as a reservoir of orbital angular momentum which potentially could stabilize and elucidate the orbital period finetuning problem remains to be investigated. It is possible that this loss of orbital angular momentum could lead to some of the intermediate systems in Fig. 2 to become solutions, rather than ending above the grey shaded region.

Another (uncertain) aspect of LMXB evolution is the effect of accretion disk instabilities (Pringle 1981; van Paradijs 1996; Lasota 2001; Dubus et al. 2001; Coriat et al. 2012). These are thermal-viscous instabilities resulting from a relatively large and sudden local increase in opacity and viscosity of the disk material at (critically) low mass-transfer rates. The high viscosity leads to a sudden outburst in which the NS accretes at a much higher rate. Outbursts are alternated by low-viscosity stages during which the disk builds up again. Stable behaviour can only persist if the entire disk has a homogeneous degree of ionization. In our work, we have partly compensated for this effect by choosing a small NS accretion efficiency (Sect. 3.2). It is possible, however, that during these outbursts (where $\left|\dot{M}_{2}\right|>\dot{M}_{\text {Edd }}$ ) some material is fed into a circumbinary disk which may affect the orbital angular momentum of the system, as mentioned above.

There is, in addition, the effect of irradiation feedback on the long-term evolution of a close-orbit binary (e.g. Büning \& Ritter 2004; Ritter 2008; Dubus et al. 1999). The impact and the modelling of this effect, leading to cyclic accretion, is still unclear and also not included in the present study. Recent work by Benvenuto et al. (2012) on the evolution of UCXBs suggests that the inclusion of irradiation feedback is not very significant for the secular evolution and thus the final properties of these systems. This is in agreement with Nelson \& Rappaport (2003) who found that the effect of excess bloating due to X-ray irradiation is small (however, see also Podsiadlowski 1991). Irradiation effects by the pulsar wind (Tavani \& Brookshaw 1992), however, possibly in combination with tidal dissipation of energy in the envelope, may cause a companion star to be thermally bloated. This may lead to evaporation and eclipses of the observed radio signals as seen in many narrow-orbit MSP systems. In the case of PSR J2051-0827 one can even measure the effects of gravitational quadrupole moment changes (Lazaridis et al. 2011), which affect the orbital evolution in a semi-cyclic and poorly understood manner that may also be applicable to close-orbit LMXBs (Applegate \& Shaham 1994; Lanza \& Rodonò 1999).

\section{Conclusions}

The main results are summarized as follows:

i) We have applied a detailed stellar evolution code to model the evolution of $\sim 400$ close binaries containing a NS and a low-mass main-sequence star. We evolved the systems to the LMXB phase with the purpose of reproducing the observed MSPs hosting He WD companions in tight orbits with $P_{\text {orb }} \simeq 2-9 \mathrm{~h}$. Using a standard prescription for orbital angular momentum losses via magnetic braking we can reproduce the observed systems for a large initial parameter space of donor star masses, NS masses, NS accretion efficiencies and magnetic braking index values.

ii) However, from an analysis of our modelling we find that a severe fine-tuning is required for the initial orbital period of the LMXBs in order to reproduce these observed systems. Based on a comparison to observational data of binary pulsars, we argue that such a fine-tuning is unlikely. We refer to this issue as the orbital period fine-tuning problem. We find further support for this problem from earlier independent studies in the literature. We conclude that something needs to be modified or is missing in the standard input physics of LMXB modelling.

iii) We have demonstrated that the $\left(M_{\mathrm{WD}}, P_{\mathrm{orb}}\right)$-relation is, in general, also valid for binary pulsars with He WDs having $P_{\text {orb }}<2$ days, although with an expected large scatter in He WD masses between $0.15-0.20 M_{\odot}$. This conclusion is based on a combination of our theoretical modelling as well as recent observational data.

Acknowledgements. A.G.I. is grateful for fruitful discussions with: Fabian Schneider, Pablo Marchant, Luca Grassitelli, Debashis Sanyal, Jean-Claude Passy and Richard Stancliffe. We thank the referee, Zhanwen Han, for helpful comments and for suggesting Fig. 10.

\section{References}

Alpar, M. A., Cheng, A. F., Ruderman, M. A., \& Shaham, J. 1982, Nature, 300, 728

Althaus, L. G., Serenelli, A. M., \& Benvenuto, O. G. 2001, MNRAS, 324, 617

Althaus, L. G., Miller Bertolami, M. M., \& Córsico, A. H. 2013, A\&A, 557, A19 Antoniadis, J., van Kerkwijk, M. H., Koester, D., et al. 2012, MNRAS, 423, 3316 Antoniadis, J., Freire, P. C. C., Wex, N., et al. 2013, Science, 340, 448

Applegate, J. H., \& Shaham, J. 1994, ApJ, 436, 312

Archibald, A. M., Stairs, I. H., Ransom, S. M., et al. 2009, Science, 324, 1411

Backer, D. C., Kulkarni, S. R., Heiles, C., Davis, M. M., \& Goss, W. M. 1982, Nature, 300, 615

Barnes, S. A. 2003, ApJ, 586, 464

Barnes, S. A., \& Kim, Y.-C. 2010, ApJ, 721, 675

Bassa, C. G., van Kerkwijk, M. H., Koester, D., \& Verbunt, F. 2006a, A\&A, 456, 295

Bassa, C. G., van Kerkwijk, M. H., \& Kulkarni, S. R. 2006b, A\&A, 450, 295 Bassa, C. G., Patruno, A., Hessels, J. W. T., et al. 2014, MNRAS, 441, 1825 Benvenuto, O. G., De Vito, M. A., \& Horvath, J. E. 2012, ApJ, 753, L33

Bhattacharya, D., \& van den Heuvel, E. P. J. 1991, Phys. Rep., 203, 1

Braun, H. 1997, Ph.D. Thesis, , Ludwig-Maximilians-Univ. München, Germany Breton, R. P., Rappaport, S. A., van Kerkwijk, M. H., \& Carter, J. A. 2012, ApJ, 748,115

Büning, A., \& Ritter, H. 2004, A\&A, 423, 281

Callanan, P. J., Garnavich, P. M., \& Koester, D. 1998, MNRAS, 298, 207

Cantiello, M., \& Langer, N. 2010, A\&A, 521, A9

Canuto, V. 1970, ApJ, 159, 641

Chen, H.-L., Chen, X., Tauris, T. M., \& Han, Z. 2013, ApJ, 775, 27

Coriat, M., Fender, R. P., \& Dubus, G. 2012, MNRAS, 424, 1991

Corongiu, A., Burgay, M., Possenti, A., et al. 2012, ApJ, 760, 100

D'Antona, F., Ventura, P., Burderi, L., et al. 2006, ApJ, 640, 950

De Vito, M. A., \& Benvenuto, O. G. 2010, MNRAS, 401, 2552

Dorch, S. B. F., \& Nordlund, A. 2001, A\&A, 365, 562

Driebe, T., Schoenberner, D., Bloecker, T., \& Herwig, F. 1998, A\&A, 339, 123

Dubus, G., Lasota, J.-P., Hameury, J.-M., \& Charles, P. 1999, MNRAS, 303, 139

Dubus, G., Hameury, J.-M., \& Lasota, J.-P. 2001, A\&A, 373, 251

Edmonds, P. D., Gilliland, R. L., Heinke, C. O., Grindlay, J. E., \& Camilo, F. 2001, ApJ, 557, L57

Eggleton, P. P. 1983, ApJ, 268, 368

Eggleton, P. P. 2001, in Evolution of Binary and Multiple Star Systems, eds. P. Podsiadlowski, S. Rappaport, A. R. King, F. D'Antona, \& L. Burderi, ASP Conf. Ser., 229, 157

Ergma, E., \& Sarna, M. J. 1996, MNRAS, 280, 1000

Faulkner, J. 1971, ApJ, 170, L99

Fedorova, A. V., \& Ergma, E. V. 1989, Ap\&SS, 151, 125

Freire, P. C. C., Wex, N., Esposito-Farèse, G., et al. 2012, MNRAS, 423, 3328

Fruchter, A. S., Stinebring, D. R., \& Taylor, J. H. 1988, Nature, 333, 237

Hubbard, W. B., \& Lampe, M. 1969, ApJS, 18, 297 
Hussain, G. A. J. 2011, in Evolution of Compact Binaries, eds. L. Schmidtobreick, M. R. Schreiber, \& C. Tappert, ASP Conf. Ser., 447, 143

Iglesias, C. A., \& Rogers, F. J. 1996, ApJ, 464, 943

Illarionov, A. F., \& Sunyaev, R. A. 1975, A\&A, 39, 185

Istrate, A. G., Tauris, T. M., Langer, N., \& Antoniadis, J. 2014, A\&A, accepted DOI: $10.1051 / 0004-6361 / 201424681$

Jacoby, B. A., Hotan, A., Bailes, M., Ord, S., \& Kulkarni, S. R. 2005, ApJ, 629, L113

Jia, K., \& Li, X. D. 2014, ApJ, accepted [arXiv: 1407. 3150]

Johnston, H. M., \& Kulkarni, S. R. 1991, ApJ, 368, 504

Joss, P. C., Rappaport, S., \& Lewis, W. 1987, ApJ, 319, 180

Kaplan, D. L., Stovall, K., Ransom, S. M., et al. 2012, ApJ, 753, 174

Kaplan, D. L., Bhalerao, V. B., van Kerkwijk, M. H., et al. 2013, ApJ, 765, 158

Kaplan, D. L., Marsh, T. R., Walker, A. N., et al. 2014, ApJ, 780, 167

Kippenhahn, R., \& Weigert, A. 1990, Stellar Structure and Evolution (Berlin: Springer)

Knigge, C., Baraffe, I., \& Patterson, J. 2011, ApJS, 194, 28

Landau, L. D., \& Lifshitz, E. M. 1971, The classical theory of fields (Oxford: Pergamon Press)

Langer, N. 1998, A\&A, 329, 551

Lanza, A. F., \& Rodonò, M. 1999, A\&A, 349, 887

Lasota, J.-P. 2001, New Astron. Rev., 45, 449

Lattimer, J. M., \& Prakash, M. 2007, Phys. Rep., 442, 109

Lazaridis, K., Wex, N., Jessner, A., et al. 2009, MNRAS, 400, 805

Lazaridis, K., Verbiest, J. P. W., Tauris, T. M., et al. 2011, MNRAS, 414, 3134

Lazarus, P., Tauris, T. M., Knispel, B., et al. 2014, MNRAS, 437, 1485

Lin, D. N. C., \& Papaloizou, J. 1979, MNRAS, 186, 799

Lin, J., Rappaport, S., Podsiadlowski, P., et al. 2011, ApJ, 732, 70

Lundgren, S. C., Zepka, A. F., \& Cordes, J. M. 1995, ApJ, 453, 419

Ma, B., \& Li, X.-D. 2009, ApJ, 691, 1611

Manchester, R. N., Hobbs, G. B., Teoh, A., \& Hobbs, M. 2005, AJ, 129, 1993

Maxted, P. F. L., Bloemen, S., Heber, U., et al. 2014, MNRAS, 437, 1681

Mestel, L. 1968, MNRAS, 138, 359

Nelemans, G. 2009, Class. Quant. Grav., 26, 094030

Nelson, L. A., \& Rappaport, S. 2003, ApJ, 598, 431

Nelson, L. A., Rappaport, S. A., \& Joss, P. C. 1986, ApJ, 304, 231

Nelson, L. A., Dubeau, E., \& MacCannell, K. A. 2004, ApJ, 616, 1124

Nice, D. J., Stairs, I. H., \& Kasian, L. E. 2008, in 40 Years of Pulsars: Millisecond Pulsars, Magnetars and More, eds. C. Bassa, Z. Wang, A. Cumming, \& V. M. Kaspi, AIP Conf. Ser., 983, 453

Paczynski, B., \& Sienkiewicz, R. 1981, ApJ, 248, L27

Panei, J. A., Althaus, L. G., Chen, X., \& Han, Z. 2007, MNRAS, 382, 779

Papitto, A., Ferrigno, C., Bozzo, E., et al. 2013, Nature, 501, 517

Parker, E. N. 1955, ApJ, 121, 491

Podsiadlowski, P. 1991, Nature, 350, 136

Podsiadlowski, P., Rappaport, S., \& Pfahl, E. D. 2002, ApJ, 565, 1107

Pringle, J. E. 1981, ARA\&A, 19, 137

Pylyser, E., \& Savonije, G. J. 1988, A\&A, 191, 57

Pylyser, E. H. P., \& Savonije, G. J. 1989, A\&A, 208, 52

Rappaport, S., Joss, P. C., \& Webbink, R. F. 1982, ApJ, 254, 616

Rappaport, S., Verbunt, F., \& Joss, P. C. 1983, ApJ, 275, 713

Rappaport, S., Podsiadlowski, P., Joss, P. C., Di Stefano, R., \& Han, Z. 1995, MNRAS, 273, 731
Refsdal, S., \& Weigert, A. 1971, A\&A, 13, 367

Ritter, H. 1988, A\&A, 202, 93

Ritter, H. 2008, New Astron. Rev., 51, 869

Roberts, M. S. E. 2013, in IAU Symp. 291, ed. J. van Leeuwen, 127

Rucinski, S. M. 1983, The Observatory, 103, 280

Sarna, M. J., Ergma, E., \& Gerškevitš-Antipova, J. 2000, MNRAS, 316, 84

Savonije, G. J. 1987, Nature, 325, 416

Schönberg, M., \& Chandrasekhar, S. 1942, ApJ, 96, 161

Serenelli, A. M., Althaus, L. G., Rohrmann, R. D., \& Benvenuto, O. G. 2002, MNRAS, 337, 1091

Shannon, R. M., Cordes, J. M., Metcalfe, T. S., et al. 2013, ApJ, 766, 5

Shao, Y., \& Li, X.-D. 2012, ApJ, 756, 85

Sills, A., Pinsonneault, M. H., \& Terndrup, D. M. 2000, ApJ, 534, 335

Skumanich, A. 1972, ApJ, 171, 565

Soberman, G. E., Phinney, E. S., \& van den Heuvel, E. P. J. 1997, A\&A, 327, 620

Spruit, H. C., \& Ritter, H. 1983, A\&A, 124, 267

Spruit, H. C., \& Taam, R. E. 2001, ApJ, 548, 900

Stappers, B. W., Bailes, M., Lyne, A. G., et al. 1996, ApJ, 465, L119

Stepien, K. 1995, MNRAS, 274, 1019

Stovall, K., Lynch, R. S., Ransom, S. M., et al. 2014, ApJ, 791, 67

Tauris, T. M. 1996, A\&A, 315, 453

Tauris, T. M. 2001, in Evolution of Binary and Multiple Star Systems, eds. P. Podsiadlowski, S. Rappaport, A. R. King, F. D’Antona, \& L. Burderi, ASP Conf. Ser., 229, 145

Tauris, T. M. 2011, in Evolution of Compact Binaries, eds. L. Schmidtobreick, M. R. Schreiber, \& C. Tappert, ASP Conf. Ser., 447, 285

Tauris, T. M., \& Savonije, G. J. 1999, A\&A, 350, 928

Tauris, T. M., \& van den Heuvel, E. P. J. 2006, in Formation and evolution of compact stellar X-ray sources (Cambridge University Press), 623

Tauris, T. M., \& van den Heuvel, E. P. J. 2014, ApJL, in press

Tauris, T. M., Langer, N., \& Kramer, M. 2011, MNRAS, 416, 2130

Tauris, T. M., Langer, N., \& Kramer, M. 2012, MNRAS, 425, 1601

Tauris, T. M., Sanyal, D., Yoon, S.-C., \& Langer, N. 2013, A\&A, 558, A39

Tavani, M., \& Brookshaw, L. 1992, Nature, 356, 320

Tutukov, A. V., Fedorova, A. V., Ergma, E. V., \& Yungelson, L. R. 1987, Soviet Astron. Lett., 13, 328

van den Heuvel, E. P. J. 1994, in Saas-Fee Advanced Course 22: Interacting Binaries, eds. S. N. Shore, M. Livio, E. P. J. van den Heuvel, H. Nussbaumer, \& A. Orr, 263

van der Sluys, M. V., Verbunt, F., \& Pols, O. R. 2005a, A\&A, 431, 647

van der Sluys, M. V., Verbunt, F., \& Pols, O. R. 2005b, A\&A, 440, 973

van Haaften, L. M., Nelemans, G., Voss, R., Wood, M. A., \& Kuijpers, J. 2012, A\&A, 537, A104

van Kerkwijk, M. H., Bassa, C. G., Jacoby, B. A., \& Jonker, P. G. 2005, in Binary Radio Pulsars, eds. F. A. Rasio, \& I. H. Stairs, ASP Conf. Ser., 328, 357

van Kerkwijk, M. H., Rappaport, S. A., Breton, R. P., et al. 2010, ApJ, 715, 51

van Paradijs, J. 1996, ApJ, 464, L139

Verbunt, F., \& Zwaan, C. 1981, A\&A, 100, L7

Vilhu, O., \& Walter, F. M. 1987, ApJ, 321, 958

Webbink, R. F., Rappaport, S., \& Savonije, G. J. 1983, ApJ, 270, 678

Weisberg, J. M., Nice, D. J., \& Taylor, J. H. 2010, ApJ, 722, 1030

Wellstein, S., \& Langer, N. 1999, A\&A, 350, 148

Wolszczan, A., \& Frail, D. A. 1992, Nature, 355, 145 